\title{
Practical Investigation of Multiband Bistatic and Monostatic RADAR SIGNATURES OF WIND TURBINES
}

Francesco Fioranelli ${ }^{1}$, Matthew Ritchie ${ }^{2}$, Alessio Balleri ${ }^{3}$, Hugh Griffiths $^{2}$

1 School of Engineering, University of Glasgow, Glasgow, UK

2 Department of Electronic and Electrical Engineering, University College London, London, UK

3 Centre for Electronic Warfare, Information and Cyber, Cranfield University, Defence Academy of the United Kingdom, Shrivenham, UK

\begin{abstract}
The negative effects wind farm clutter has on the performance of radar systems for Air Traffic Control and Air Surveillance is well-known in the radar research community and several mitigation techniques have been proposed to address this problem. These include bistatic and multistatic radar systems providing multiple views of the area under surveillance, and hence potential additional information that can be used to improve the receiver performance. This paper presents the analysis of a set of experimental data collected simultaneously by two radar systems, one operating at S-band and one at X-band, of echoes from an operational wind farm in the UK near Oxford. This analysis presents several parameters extracted from the time domain data and the Doppler spectra, such as Doppler centroid and bandwidth of the micro-Doppler signature as well as amplitude statistics of the time domain returns. These parameters are characterised using data recorded at monostatic and bistatic nodes, as well as at different polarisation combinations.
\end{abstract}

\section{Introduction}

Reducing the dependency on fossil fuels and the related emissions of greenhouse gases is a key priority of many Western and developing countries, achievable with an increasing amount of electricity generated from environmental-friendly and renewable sources such as solar and wind energy. The UK Government has set the objective of producing $15 \%$ of energy from renewable sources by 2020, and onshore and offshore wind energy is thought to provide a significant contribution to achieve this target [1]. For example, it has been estimated by RenewableUK, the leading trade association for renewable energy producers in the UK, that the total onshore capacity in the UK has produced approximately $17 \mathrm{TWh}$ at the end of 2014, which is the equivalent consumption of more than 4 million households [2]. They have also calculated that a single 2.5 MW wind turbine can produce enough energy to support 1400 households in a year, equivalent to running an average PC for over 2000 years or making 230 million cups of tea [2]. 
Besides the criticism related to the impact on local communities and to the cost of operating and maintaining onshore and offshore wind farms, their development plans often face 'radar objections'. These are related to the impact of wind farms on radar systems used for crucial functions, such as Air Traffic Control (ATC) for civilian aircraft and Air Defence radar systems to detect and localise potential unauthorised and hostile aircraft. The development of wind farms can therefore be hindered by objections raised by civilian and military bodies operating these radar systems, resulting in delays, complications at the planning phase, or even a complete block of the proposed development. It has been reported for instance that more than $50 \%$ of the UK developments have faced 'radar objections' from the Ministry of Defence or from the aviation sector, accounting for approximately $12 \mathrm{GW}$ of capacity in 2013 [3-4]. Adverse effects wind turbines have on radar systems include increased undesired echoes from the rotating turbine blades which may generate false alarms, obscure actual targets hence reducing the probability of detection for targets located in the space above and around the wind farm, often referred to as 'desensitisation of the radar', and worsened tracking and plotting capabilities for targets in these affected areas caused by saturation of the tracker due to many false echoes [5]. Typical wind turbines have very large Radar Cross Section (RCS) compared with conventional air targets, as much as $55 \mathrm{dBsm}$ of peak RCS against around 0-10 dBsm for small fighter jets, and the Doppler shifts caused by the fast-moving tips of the turbine blades can be comparable to those produced by slow moving air targets such as small aircrafts, landing commercial aircrafts, and helicopters [5].

Many varied techniques have been suggested in the literature to address this problem and mitigate the aforementioned adverse effects, with a good summary of the main approaches in [6] and in the document issued by the UK Civil Aviation Authority (CAA) 'Policy and Guidelines on Wind Turbines' [7]. Some of these techniques involve modifications of the design of the actual turbines, e.g. terrain screening, relocation and rearranging the position of some turbines, reshaping of parts of the turbines to reduce the occurrence of specular electromagnetic reflections, and employing Radar Absorbing Material (RAM) to achieve an overall reduction of RCS. Although these techniques can be effective, they increase the development costs for the wind farm and may reduce the energy production capacity, thus compromising their suitability. Other approaches act on the radar system affected by the wind farm, trying to improve its performance. These include adapting antenna beams with tilting and beam-forming to minimise the undesired returns from the turbines, improving the detection and tracking algorithms to remove the contribution from the wind farm, and employing multiple sensors with the so-called 'in-fill radar' to complement the coverage of the primary radar.

The possibility of modelling the RCS and Doppler signatures of various models of wind turbines is important to predict the effect of these structures on radar systems. Significant research has been 
carried out to develop accurate and computationally efficient models of RCS [8-14], and to measure scaled models of turbines in controlled setups to investigate the effect of different yaw angles and rotation speeds on the resulting radar signature [15-16]. The performance of realistic radar systems affected by wind farm clutter has also been characterised, for example for the effectiveness of Ground Moving Target Indication (GMTI) algorithms for targets within the wind farm area [17], for the behaviour of weather radar stations [18-20], for maritime radar systems [21-22], and for the detection and tracking of unauthorised aircraft entering restricted zones [23]. A different approach aims to develop totally new designs for wind farms to replace the conventional horizontal axis turbines with 3 blades, for instance vertical axis turbines such as those simulated and tested with a scaled model in [24], or wind lens turbines developed and tested experimentally in [25]. In [26], partial reshaping of the nacelle design combined with the application of RAM material has been considered to reduce the overall RCS, by taking also into account the costs of this intervention. In [27] RAM material has been used within the design of a lighting protection system for the turbine, and numerical simulations have been provided to evaluate the RCS reduction effect. Digital signal processing algorithms specifically designed to mitigate the impact of wind farm clutter on radar systems have been also proposed [2830].

In general, limited research has been published analysing experimental data from operational wind turbines. The US Air Force Research Laboratory has carried out a comprehensive experimental campaign covering four radar bands from L-band to X-band [31-32]. They have analysed the RCS values and the Doppler signatures, and have shown that a good agreement could be achieved between experimental data and data simulated with a numerical model based on the software X-patch and CAD models of the turbines. Additional experimental data have been shown by the authors in [33], with wind turbine signatures recorded by a weather surveillance radar and interpolation algorithms to reduce the clutter by losing some spatial resolution. In [34], statistical models of RCS for the returns from the turbines extracted from the data collected in [31-32] have been presented and detection probabilities assuming Swerling-1 models for targets, showing that the T-Location Scale distribution provided the best fit to describe this type of clutter. In [35], the authors of that work presented experimental results from a campaign to characterise the RCS of different models of operational turbines in South Africa at VV and $\mathrm{HH}$ polarisations and S-band and L-band frequencies, highlighting the differences due to the variety of aspect angles and the structural variations of turbines manufactured by different companies. In [36] the authors presented preliminary results related to wind farm backscattering collected by a polarimetric radar system operating at S-band. In [37] measurements at short-range of operational wind turbines collected by C-band and K-band radar 
prototypes were presented, showing good agreement with a proposed numerical model and aiming to use the generated results for monitoring the behaviour of the turbines.

Bistatic and multistatic surveillance radar systems which can exploit a network of multiple transmitters and receivers have also been previously proposed to mitigate wind farm clutter [7]. The additional degrees of freedom provided by multiple radar nodes in choosing different radar parameters in different operational conditions, and the multi-perspective view on the area under surveillance and the spatial diversity of multiple nodes to be deployed may provide some advantages resulting in lower clutter Doppler spread and lower radar clutter-to-target cross section. To the best of our knowledge, there is very little published data regarding wind farm signatures collected by multistatic radar systems. This paper presents the analysis of simultaneous monostatic and bistatic radar signatures of operational wind turbines collected by the multistatic S-band radar NetRAD developed at University College London (UCL) in January 2015. A X-band radar was also simultaneously deployed by Cranfield University at the location of the NetRAD monostatic transceiver for multiband data comparison. The analysis presented within this article expands the preliminary empirical results presented in our previous conference paper [38], by extracting from the same dataset additional quantitative parameters to characterise the radar signatures for different bistatic angles and polarisations, such as the Doppler centroid and the Doppler bandwidth of the micro-Doppler signatures, and the amplitude statistics of the signatures in the time domain. Some of these parameters were investigated for a different dataset (May 2015) in [39-40], and it is believed that the comprehensive analysis provided in this manuscript can help better characterise and understand bistatic wind farm signatures, given the lack of experimental data in this context and the possibility of comparing simultaneously collected monostatic and bistatic data given by these data.

The remainder of this paper is organised as follows. Section 2 describes the radar systems and the experimental setup used for data collection. Section 3 presents the data analysis and the parameters extracted from the wind turbine signatures for monostatic and bistatic data at S-band and at X-band, with different polarisations. Section 4 finally concludes the paper.

\section{Experimental setup and radar systems}

The data analysed in this paper were collected in a series of experiments in January 2015 at the Westmill Wind Farm in Watchfield, near Oxford, outside the perimeter of the Defence Academy of the UK. This wind farm consists of five $49 \mathrm{~m}$ tall turbines, each one with three $31 \mathrm{~m}$ long blades. Unfortunately, the ground truth data on the blades rotation velocity and the wind speed recorded at the nacelle of each turbine are not made publicly available from the management of the wind farm, so it is 
not possible to provide wind speed and direction for the specific time and day of the experiment. The average wind speed on the day was retrieved from historical data of nearby weather stations and equal to approximately $4 \mathrm{~m} / \mathrm{s}$, but this is only an approximation of the actual wind speed at the top of each turbine. Information on the yaw angle, i.e. the azimuth orientation of the blades rotation plane for each turbine and how this changes dynamically with wind conditions, is not available and can only be empirically estimated by visual inspection of the turbines.

A diagram of the geometry of the experimental setup is shown in Fig. 1. The position of the two radar systems used to collect the data are shown, relative to the wind turbine targets. The first radar is the Sband multistatic pulsed coherent radar NetRAD developed at UCL and consists of three identical and separable radar nodes [41]. This system used $+23 \mathrm{dBm}$ transmitted power, $0.6 \mu$ s pulse length, $45 \mathrm{MHz}$ bandwidth for the linear up-chirp modulation, and $5 \mathrm{kHz}$ Pulse Repetition Frequency (PRF). This was sufficient to include the whole micro-Doppler signatures in the unambiguous Doppler region, and $10 \mathrm{~s}$ duration for each recording. The antenna beam-widths were approximately $10^{\circ}$ in azimuth and elevation, and the gain was $24 \mathrm{dBi}$. The second radar system is a monostatic X-band Continuous Wave (CW) radar system developed at Cranfield University. The radar transmits a continuous unmodulated waveform with a power of approximately $+15 \mathrm{dBm}$ at $10 \mathrm{GHz}$. The system samples both the I and $\mathrm{Q}$ components of the received signal digitised with a TiPie HS4 oscilloscope at $10 \mathrm{kHz}$ sampling rate and 12-bit resolution. The transmitting and receiving antennas used for the experiment were two identical horn antennas, separated of approximately $25 \mathrm{~cm}$, with a gain of $20 \mathrm{dBi}$ and a $15^{\circ} \times 20^{\circ}$ beam-width.

Two NetRAD nodes, with a baseline separation of approximately $50 \mathrm{~m}$, were deployed for this experiment as shown in Fig. 1. The X-band radar was located at the position of the monostatic NetRAD transceiver to record simultaneous data and allow a comparison of multi-band data. Monostatic and bistatic S-band data were collected simultaneously thanks to a common trigger and clock reference signal used to synchronised the nodes. The X-band data may present a small delay of fractions of seconds due to the non-perfect manual synchronisation of the start time for both radar systems. This difference is practically not significant in the processing, as multiple rotation cycles of the turbines were recorded in each dataset to capture the average behaviour and compare the two systems. The separation between the nearest turbine and the baseline was approximately $432 \mathrm{~m}$, generating a bistatic angle of about $6.5^{\circ}$, whereas the separation between the second turbine and the baseline was approximately $616 \mathrm{~m}$, with a bistatic angle of $4.6^{\circ}$. These turbines will be labelled as Turbine Under Test 1 and 2 (TUT1 and TUT2), respectively, and are shown in the map view part of Fig. 1. The data for each TUT were collected separately, by aiming the antennas to the nacelle of the specific turbine using optical rifle-scopes. Data for different polarisations were collected to investigate the effect of polarisation on the radar signatures, namely VV (co-polarised vertical-vertical), HH (co- 
polarised horizontal-horizontal), and VH (cross-polarised vertical-horizontal). The change of polarisation and the change of turbine under test required a manual rotation of the antennas, hence the data collected for different polarisations or different turbines are not simultaneous, and the time interval between groups of measurements with different polarisations is approximately 20 minutes

In previous work [42] the transmitter location was limited to one of the two nodes deployed. As part of this analysis some data was gathered using the transmitting capabilities from the bistatic radar node as well. This allows for a direct comparison of a monostatic capture to a bistatic capture taken at the same geographic location. These measurements and data will be referred to as 'reciprocal' in the remainder of this paper, and the roles of the S-band nodes for 'reciprocal' and normal measurements is graphically indicated in Fig. 1 for more clarity. As performing 'reciprocal' measurements involved a manual change of the connection of the RF cables from the antenna to the radar node, normal and 'reciprocal' measurements are separated in time by approximately 2 to 5 minutes.

It should also be noted that the data collection was performed in the near-field of the wind turbines. The minimum far-field distance necessary for using plane wave illumination conditions for these measurements would be in the order of $15 \mathrm{~km}$, according to the usual far-field relation $2 D^{2} / \lambda$ and considering the $12.5 \mathrm{~cm}$ wavelength at $\mathrm{S}$-band and the $31 \mathrm{~m}$ blade length. This distance would increase up to approximately $64 \mathrm{~km}$ at X-band. Far-field measurements appear to be therefore infeasible with the available radar systems. This issue was also mentioned in [15, 43], arguing that it is not unlikely that wind farms, especially those with large and tall turbines, can be located in the near-field of practical radar systems and therefore the analysis of near-field data is still significant and reasonable.
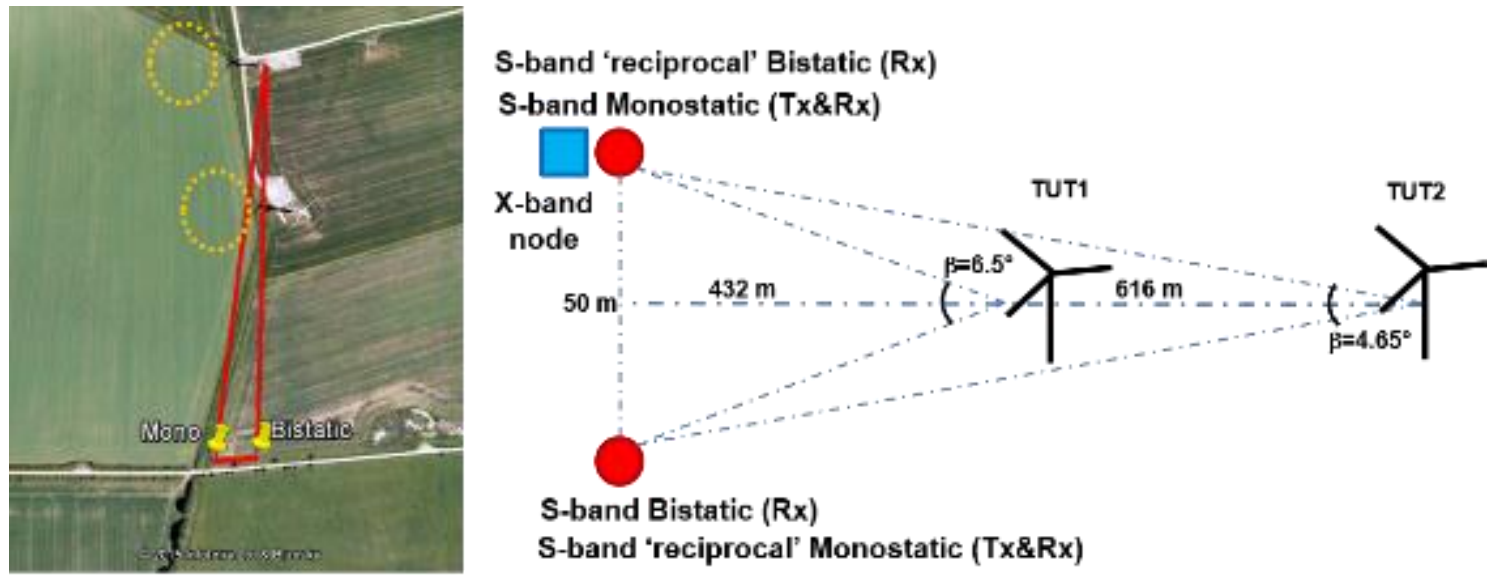

Figure 1 Sketch and top view of the experimental setup with S-band and X-band radar deployed and the turbines

\section{Data Analysis}

This section describes the analysis performed on the data and discusses the results. The S-band radar data were processed to obtain Range Time Intensity (RTI) plots in which the different turbines under 
test can be separated, as the S-band radar has sufficient range resolution to record the returns of the different turbines at different range bins. Then the micro-Doppler signatures were extracted for each turbine independently from the others by applying Short Time Fourier Transform (STFT) on the relevant range bins for each turbine. The returned echoes to the $\mathrm{CW} \mathrm{X}$-band radar were in practice dominated by the first TUT, although some of the contributions of the second turbine are at times present in the micro-Doppler signature. When present, the TUT2 returns cannot be distinguished from the TUT1 returns, as this type of radar cannot resolve targets in the range domain. The micro-Doppler processing for the S-band data was performed using STFT with Hamming window of $0.6 \mathrm{~s}$ duration and $95 \%$ overlap. About one quarter of this duration was used to generate the spectrograms at X-band. As an example, Fig. 2 shows the normalised RTI plots for S-band monostatic and bistatic data collected in $\mathrm{HH}$ polarisation for 5 seconds. The turbines appear as vertical spaced lines at a certain two-way range from the radar indicated in the $\mathrm{X}$-axis, where the return of each turbine is located at different range bins.

(a)

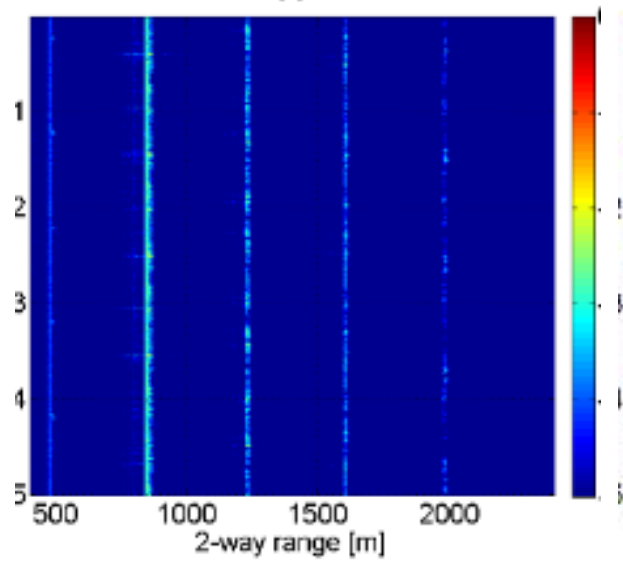

(b)

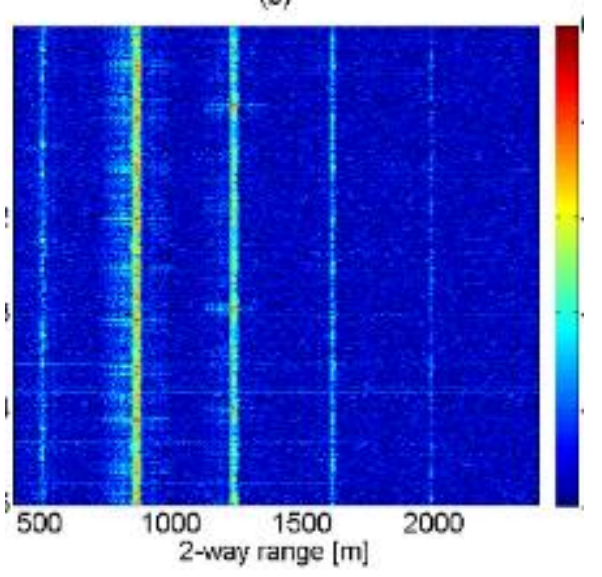

Figure 2 RTI plots for monostatic (a) and bistatic (b) HH polarised data collected at S-band

\subsection{S-band data}

The spectrograms of the monostatic and bistatic data for different polarisations are presented in Figs. 3-5 for an empirical characterisation of the micro-Doppler signatures. The spread of the monostatic signature appears to be broader in Doppler than the corresponding bistatic signature for $\mathrm{HH}$ polarised TUT1 data (Fig. 3a and 3b). The signature appears to be more symmetric in the case of the TUT2 (Fig. $3 \mathrm{c}$ and $3 \mathrm{~d}$ ), whereas the negative Doppler component seems to dominate the positive one for data related to the TUT1. Looking at VV polarised data in Fig. 4, it would appear that the monostatic signature presents a higher return for the TUT1, but there is overall more symmetry with respect to the corresponding $\mathrm{HH}$ dataset, whereas the data related to the TUT2 in Fig. 4c and 4d appear to present comparable returns. The positive Doppler blade flashes seem to have on average higher intensity than 
the negative Doppler flashes for VV data, and this effect is more evident for TUT2 data for which the blade flashes appear to be practically below the noise floor of the measurements. It is believed that the difference between the simultaneous signatures of the two turbine under tests can be related to the different aspect angles with respect to the line-of-sight of the radar nodes, and this angle changes dynamically with time as the turbines react to changes in wind speed and direction and change their yaw angle to maximise the energy production. Unfortunately, actual real-time data on the variations of this angle could not be obtained from the wind farm operator, thus only empirical considerations can be made on the effect of this parameter when comparing different datasets. The VH cross-polarised signatures are shown for completeness in Fig. 5. As expected the overall signature intensity is much lower than for co-polarised data, and the blade flashes are visible for the data related to the TUT1, whereas the signature is very weak for the TUT2.
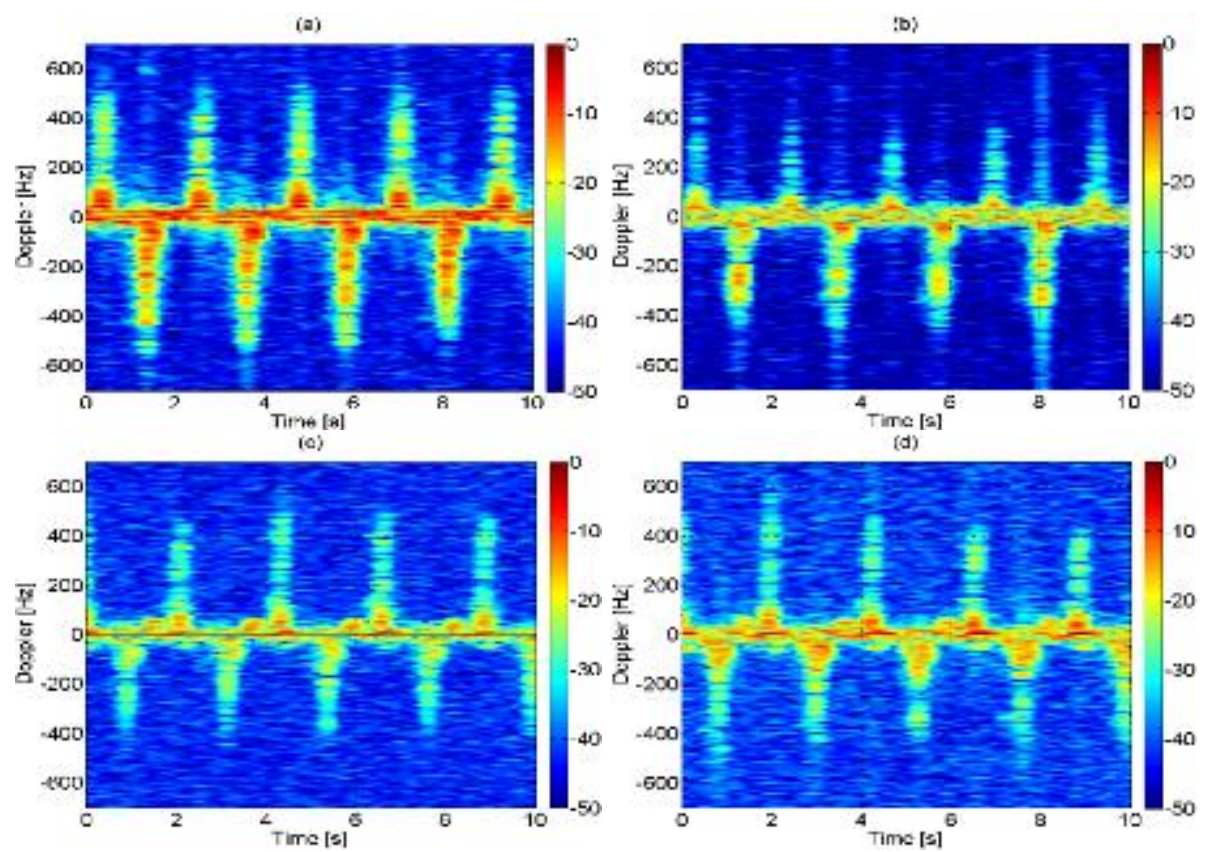

Figure 3 S-band spectrograms for HH polarization: TUT1 data for monostatic (a) and bistatic (b) radar node, and TUT2 data for monostatic (c) and bistatic (d) radar node 

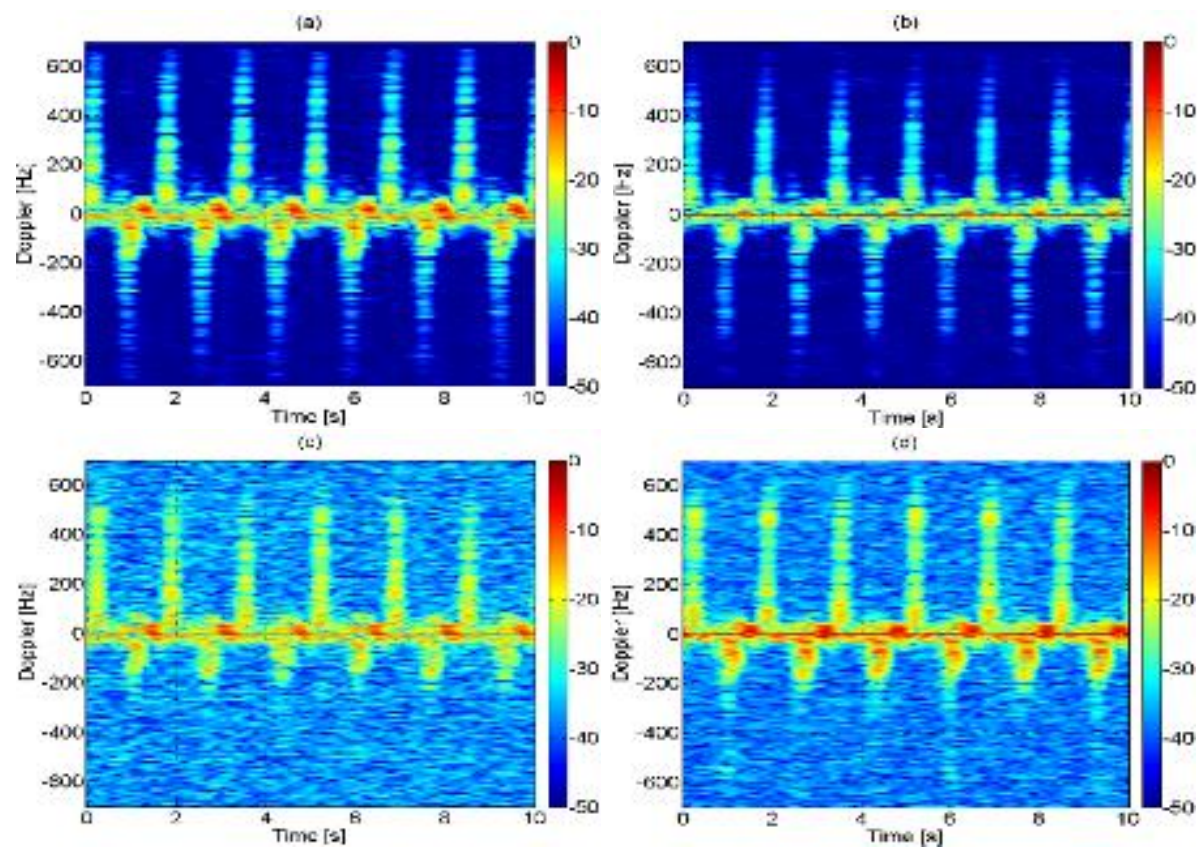

Figure 4 S-band spectrograms for VV polarization: TUT1 data for monostatic (a) and bistatic (b) radar node, and TUT2 data for monostatic (c) and bistatic (d) radar node
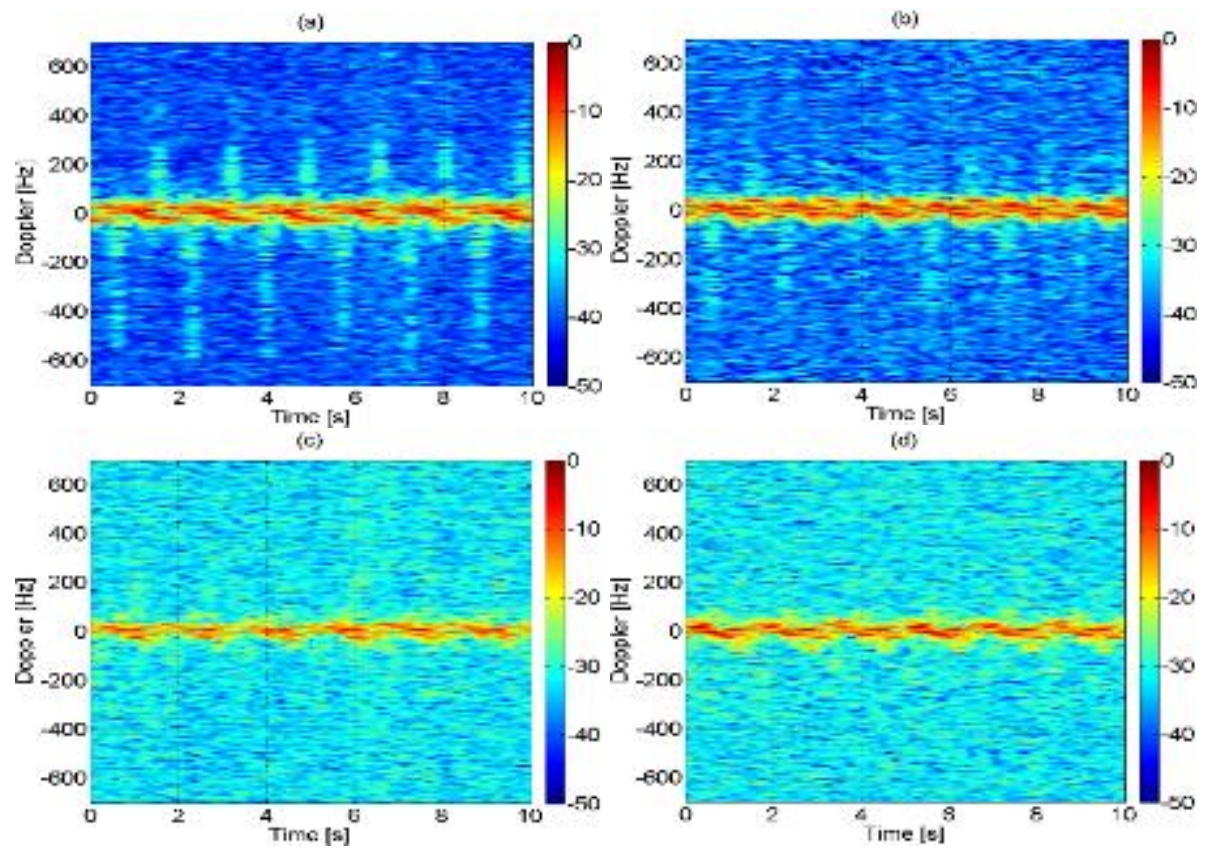

Figure 5 S-band spectrograms for VH polarization: TUT1 data for monostatic (a) and bistatic (b) radar node, and TUT2 data for monostatic (c) and bistatic (d) radar node

Quantitative parameters have been extracted from the signatures to compare the simultaneous monostatic and bistatic data. The first couple of parameters are the Doppler centroid and the bandwidth around the centroid. The former estimates the centre of gravity of the spectrogram, and the latter aims to estimate the signature bandwidth around this centre of mass. An advantage of these parameters is their independence from any normalisation, so they can be applied also to non-calibrated data without losing information. These parameters have been calculated as in equation (1) and (2), where $S(i, j)$ is 
the spectrogram at the $i^{\text {th }}$ Doppler bin and $j^{\text {th }}$ time bin, and $f_{C}$ is the Doppler centroid, $B_{C}$ the Bandwidth centroid [25].

$$
\begin{gathered}
f_{c}(j)=\frac{\sum_{i} f(i) S(i, j)}{\sum_{i} S(i, j)} \\
B_{c}(j)=\sqrt{\frac{\sum_{i}\left(f(i)-f_{c}(j)\right)^{2} S(i, j)}{\sum_{i} S(i, j)}}
\end{gathered}
$$

The centroid and bandwidth values are shown in Figs. 6-8 for different turbines and polarisations, comparing the results from simultaneous monostatic and bistatic data. The results appear to be similar for both $\mathrm{HH}$ and VV co-polarised data. The centroid values are comparable for monostatic and bistatic data, with the positive and negative Doppler peaks corresponding to the blade flashes while the turbine under test is rotating. These peaks appear to be larger at the positive Doppler values for the monostatic data in case of the TUT1 (Figs. 6a and 7a), and of comparable values for the TUT2 and at the negative Doppler values for the TUT1. More significant variations between simultaneous monostatic and bistatic data can be seen for the bandwidth parameters. For the TUT2 (Figs. 6d and 7d) there are significant differences with higher bandwidth for the monostatic signatures, whereas for the TUT1 these differences are less significant but on average the monostatic data appear to have larger values than the corresponding bistatic. Only the centroid and bandwidth related to the TUT1 are shown for cross-polarised VH data in Fig. 8, as the micro-Doppler signature had a significantly reduced SNR and feature extraction was not feasible. It should be noticed that the centroid has much smaller values compared with the co-polarised data as the signature is overall weaker. The values of the bandwidth appear to be higher for the monostatic case compared with the simultaneous bistatic.

These results appear to suggest that the different operational parameters such as the polarisation, the position of the radar nodes with respect to the wind farm, and the turbine yaw angle with respect to the line-of-sight of the radar nodes can have a significant impact on the wind turbine signatures, and thus on the level of clutter recorded at each radar node. Considering in perspective a multistatic radar system with different nodes, the operator can potentially deploy them in such a way as to take advantage of the presented clutter diversity effect, depending on the aforementioned operational parameters in order to minimise the recorded clutter and its impact, taking into account that the yaw angle of each turbine cannot be controlled and changes dynamically with wind conditions.

The results in Figs. 6-8 appear to suggest that the bistatic node recorded clutter with smaller bandwidth, i.e. signature less spread out in Doppler, at least at the limited bistatic angle values achieved for the dataset analysed in this work $\left(6.5^{\circ}\right.$ and $\left.4.65^{\circ}\right)$. The analysis of other data collected at a different time with larger bistatic angles $\left(23^{\circ}\right.$ and $\left.13^{\circ}\right)$ in [40] provided comparable results in some 
cases and opposite results in other cases (i.e. larger Doppler bandwidth for bistatic data compared with monostatic). These experiments appear to confirm the impact of polarisations and bistatic angles on the intensity and Doppler spread of wind farm clutter, but more experimental data are needed to fully characterise this behaviour, despite the challenge of collecting the required large amount of data to account for the variability of all the parameters involved.

(a)

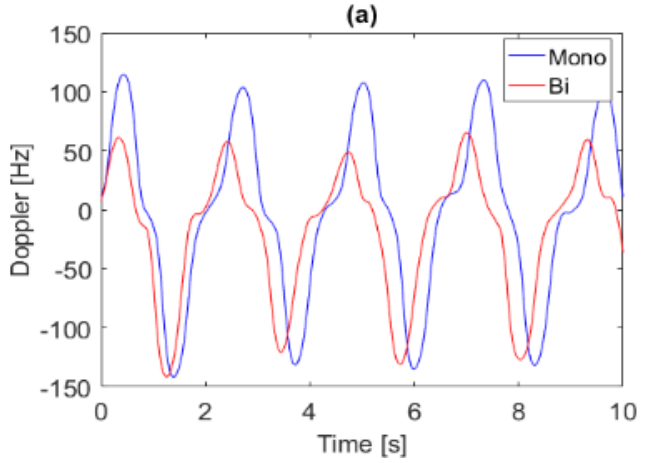

(c)

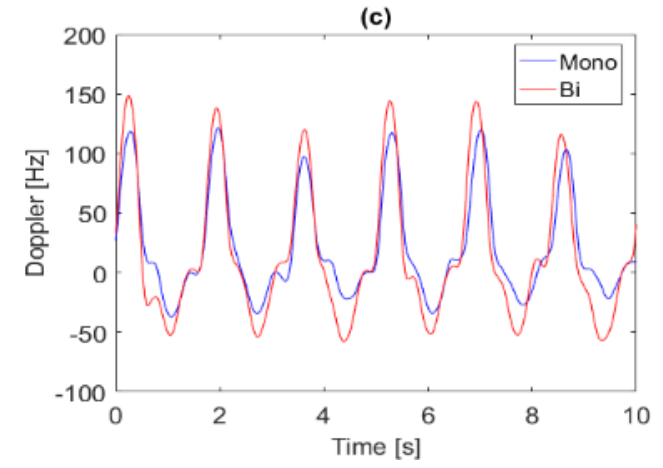

(b)

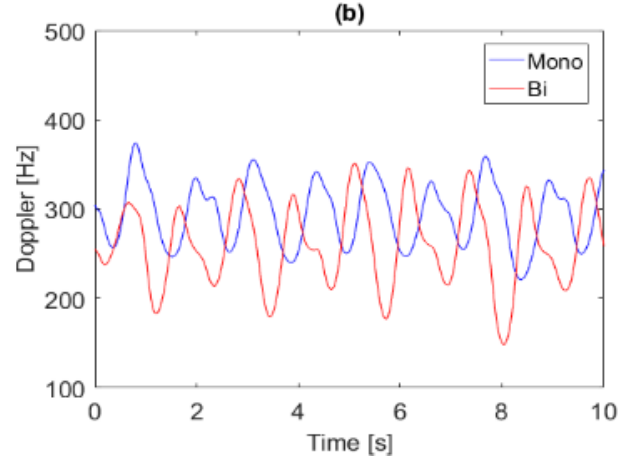

(d)

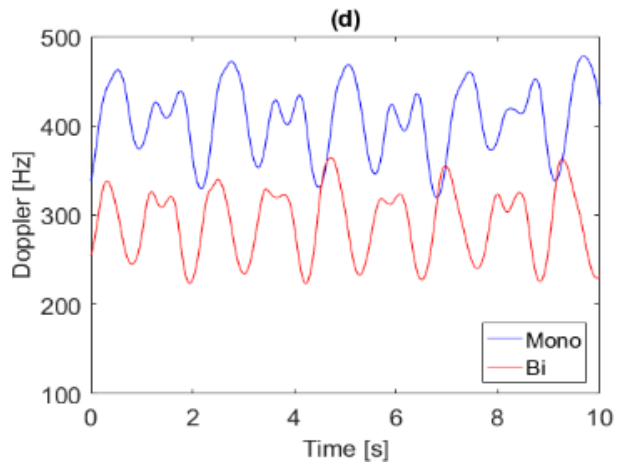

Figure 6 Monostatic vs bistatic Doppler centroid (a) and bandwidth (b) for TUT1, and Doppler centroid (c) and bandwidth (d) for TUT2 - HH polarised S-band data 
(a)

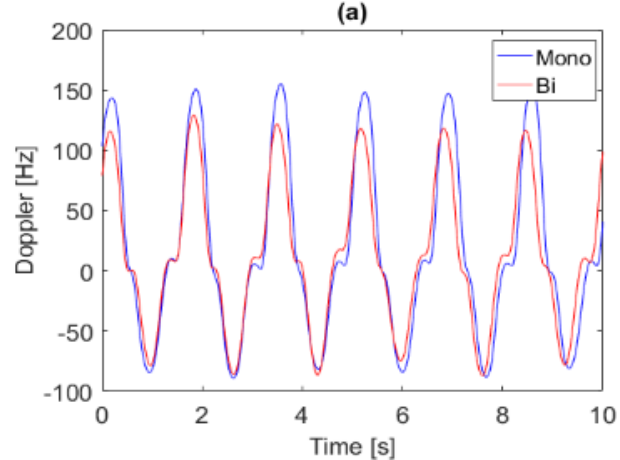

(c)

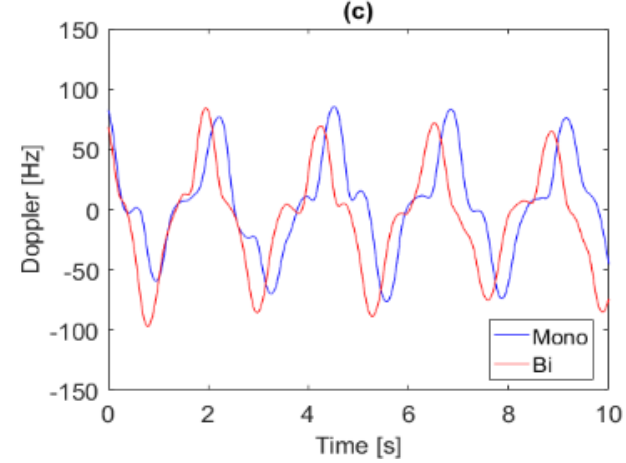

(b)

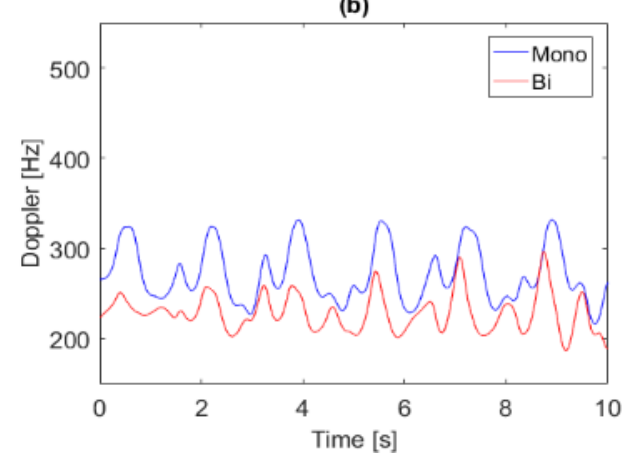

(d)

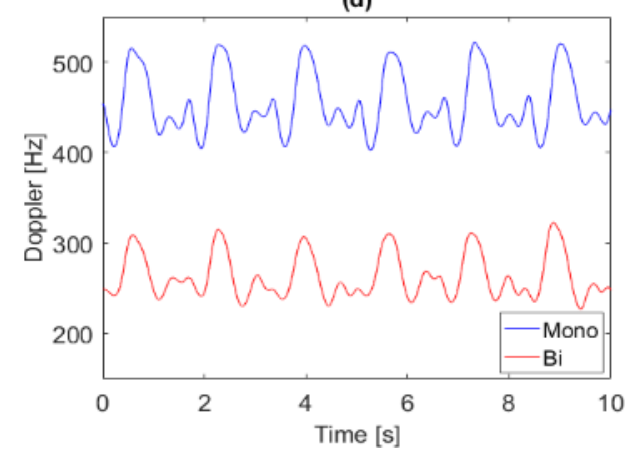

Figure 7 Monostatic vs bistatic Doppler centroid (a) and bandwidth (b) for TUT1, and Doppler centroid (c) and bandwidth (d) for TUT2 - VV polarised S-band data
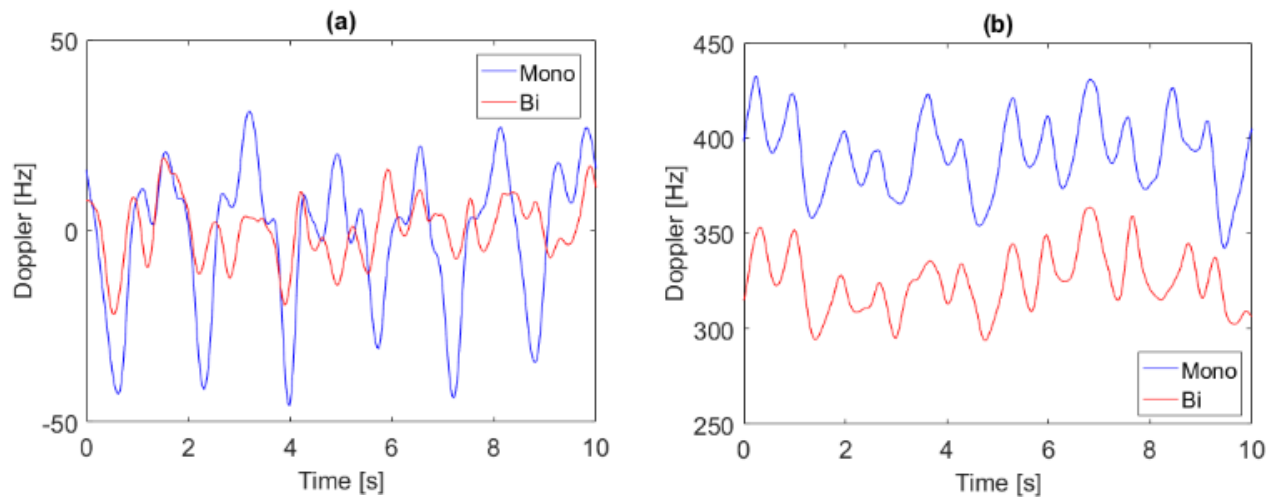

Figure 8 Monostatic vs bistatic Doppler centroid (a) and bandwidth (b) for TUT1 - VH polarised S-band data

A key parameter that has also been considered for a monostatic vs bistatic quantitative comparison is the amplitude statistics of the radar returns from the wind turbines, and their fittings to different theoretical distributions, as statistical analysis takes into account the dynamic changes in aspect angle of the turbine rotation plane with respect to the radar nodes [34, 39]. In this work seven statistical distributions have been fitted using the Maximum Likelihood Estimation (MLE) method to the intensity samples collected for different turbines under test and polarisations, namely the Burr distributions, Log-logistic, Log-Normal, T-Location Scale, Rayleigh, Weibull, and Gamma distributions. The best fit has been evaluated by calculating the Root Mean Square Error (RMSE) in the logarithmic domain between the theoretical distributions and the cumulative distribution function of the actual data. The resulting RMSE values are summarised in Table 1 for each polarisation and 
turbine under test considered, indicating the error for each radar node (transmitter and receiver) and the minimum error obtained across different distributions in bold font for each case. The T-Location Scale distribution appears to provide the best fit, with the lowest RMSE in the majority of the cases considered. This is in agreement with previous studies on actual radar data of operational wind turbines reported in $[34,39]$. Fig. 9 presents the Probability Distribution Function (PDF) of the bestfitted T-Location Scale and the histograms of the corresponding data for the different polarisations and turbines, showing a good fit especially for the tail of the distribution across the considered datasets. It should be noted that the horizontal axis for the figures referring to the TUT2 has been limited as the intensity values are in general smaller because this turbine is further away from the radar than the TUT1. It is interesting to observe that in all cases the return from the bistatic node (node 1) seems to have higher mean and longer tails compared with the simultaneous monostatic data. This appears to be in contrast with the analysis of some datasets collected with larger bistatic angles in [39], where bistatic data had shorter tails. The empirical CDFs and the CDFs estimated from the T-Location scale distributions are also reported for completeness in Fig. 9, for the three HH, VV, VH polarisations cases of the TUT1. Additional experimental data are needed to provide a univocal conclusion regarding the comparison monostatic vs bistatic data as a function of bistatic angles and polarisations, but clear differences in the intensity amplitude statistics can be observed in these data. For completeness, the equation for the PDF of the T-Location Scale is reported in (3), where $\sigma$ is the scale parameter (width of the distribution), $\mu$ is the location parameter (mean value of the samples), $v$ is the number of degrees of freedom (which is related to the distribution tail, with low values of $v$ implying heavier and longer tails), and $\Gamma$ is the Gamma function. Table 2 shows the parameters of the T-Location scale distribution for the different polarisations and turbines under test considered in this work. Parameters for the Weibull distribution are also shown as it appears to be visually similar to the empirical data. The distributions parameters exhibit some variability with operational parameters concerning the radar such as polarisation, turbine under test, and monostatic/bistatic node. This variability was also observed for a different dataset collected for the same wind farm and radar system, but in a different configuration and different season [39]. Future work will consider longer recordings for better statistical characterisation, as well as acquire ground truth data on wind speed and direction and yaw angle of turbines to relate any operational and environmental change to changes in the distribution parameters. This can in turn feed into better modelling of wind farm clutter in different conditions.

$$
f_{X}(x, \mu, \sigma, v)=\frac{\Gamma\left(\frac{v+1}{2}\right)}{\sigma \sqrt{v \pi} \Gamma\left(\frac{v}{2}\right)}\left(1+\frac{\left(\frac{x-\mu}{\sigma}\right)^{2}}{v}\right)^{-\frac{v+1}{v}}
$$


Table 1 RMSE values for fittings of radar data to different theoretical distributions

\begin{tabular}{|c|c|c|c|c|c|c|c|c|c|c|c|c|}
\hline \multirow{2}{*}{ RMSE } & \multicolumn{2}{|c|}{ HH TUT 1} & \multicolumn{2}{|c|}{ HH TUT 2} & \multicolumn{2}{|c|}{ VV TUT 1} & \multicolumn{2}{|c|}{ VV TUT 2} & \multicolumn{2}{|c|}{ VH TUT 1} & \multicolumn{2}{|c|}{ VH TUT 2} \\
\hline & Mono & $\mathbf{B i}$ & Mono & $\mathbf{B i}$ & Mono & $\mathbf{B i}$ & Mono & $\mathbf{B i}$ & Mono & $\mathbf{B i}$ & Mono & $\mathbf{B i}$ \\
\hline Burr & 1.55 & 1.55 & 1.73 & 1.55 & 1.99 & 2.73 & 1.61 & 1.80 & 1.80 & 2.39 & 1.18 & 1.89 \\
\hline Log-logistic & 1.50 & 1.45 & 1.71 & 1.49 & 2.03 & 2.87 & 1.40 & 1.82 & 1.81 & 2.51 & 1.18 & 1.89 \\
\hline Log-normal & 1.54 & 1.49 & 1.78 & 1.54 & 2.11 & 2.96 & 1.48 & 1.88 & 1.85 & 2.54 & 1.19 & 1.95 \\
\hline T-Location Scale & 1.41 & 1.46 & 1.53 & 1.43 & 1.63 & 2.36 & 1.67 & 1.50 & 1.58 & 2.17 & 1.16 & 1.67 \\
\hline Rayleigh & 1.84 & 1.79 & 1.86 & 1.81 & 2.20 & 2.27 & 1.79 & 2.12 & 2.05 & 2.46 & 1.80 & 2.02 \\
\hline Weibull & 1.45 & 1.50 & 1.66 & 1.48 & 1.85 & 2.58 & 1.56 & 1.70 & 1.74 & 2.39 & 1.04 & 1.83 \\
\hline Gamma & 1.55 & 1.55 & 1.75 & 1.56 & 2.00 & 2.80 & 1.63 & 1.81 & 1.82 & 2.43 & 1.18 & 1.90 \\
\hline
\end{tabular}

Table 2 Parameters of the T-Location scale and Weibull distributions fitted to the different data considered here

\begin{tabular}{|c|c|c|c|c|c|c|}
\hline Parameters & Nodes & T-Loc Mu & T-Loc Sigma & T-Loc Nu & Weibull scale A & Weibull shape B \\
\hline \multirow{2}{*}{ HH TUT 1 } & Mono & 0.0131 & 0.0064 & 3.3142 & 0.0166 & 1.5624 \\
\cline { 2 - 7 } & $\mathbf{B i}$ & 0.0180 & 0.0095 & 5.3555 & 0.0218 & 1.6551 \\
\hline \multirow{2}{*}{ HH TUT 2 } & Mono & 0.0063 & 0.0029 & 3.7611 & 0.0078 & 1.7640 \\
\cline { 2 - 7 } & $\mathbf{B i}$ & 0.0105 & 0.0053 & 3.9284 & 0.0131 & 1.6173 \\
\hline \multirow{2}{*}{ VV TUT 1 } & $\mathbf{M o n o}$ & 0.0204 & 0.0097 & 3.3112 & 0.0260 & 1.6817 \\
\cline { 2 - 7 } & $\mathbf{B i}$ & 0.0360 & 0.0143 & 8.4900 & 0.0414 & 2.3161 \\
\hline \multirow{2}{*}{ VV TUT 2 } & Mono & 0.0033 & 0.0016 & 3.8237 & 0.0041 & 1.5556 \\
\cline { 2 - 7 } & $\mathbf{B i}$ & 0.0102 & 0.0051 & 3.1780 & 0.0131 & 1.6140 \\
\hline \multirow{2}{*}{ VH TUT 1 } & Mono & 0.0072 & 0.0037 & 4.4818 & 0.0088 & 1.6935 \\
\cline { 2 - 7 } & $\mathbf{B i}$ & 0.0100 & 0.0052 & 27.1074 & 0.0114 & 1.9369 \\
\hline \multirow{2}{*}{ VH TUT 2 } & $\mathbf{M o n o}$ & 0.0026 & 0.0012 & 1.7128 & 0.0038 & 1.1816 \\
\cline { 2 - 7 } & $\mathbf{B i}$ & 0.0053 & 0.0026 & 5.9275 & 0.0063 & 1.8032 \\
\hline
\end{tabular}


(a)

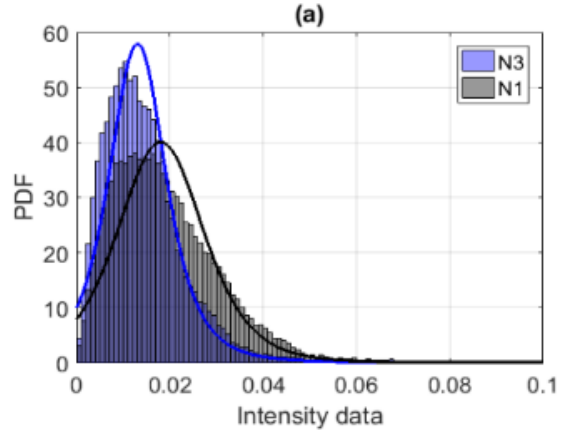

(c)

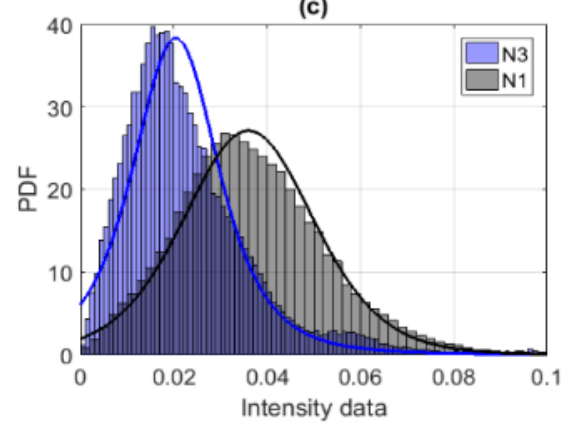

(e)

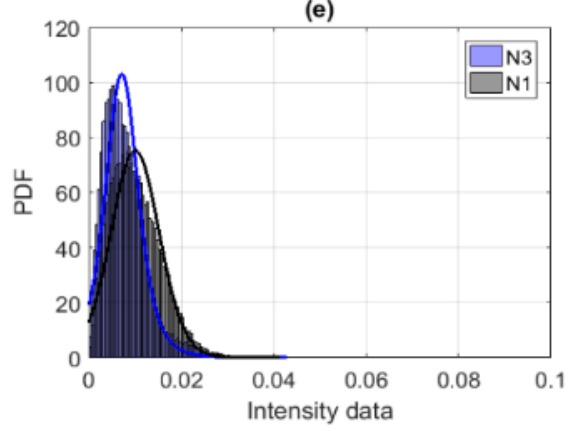

(b)

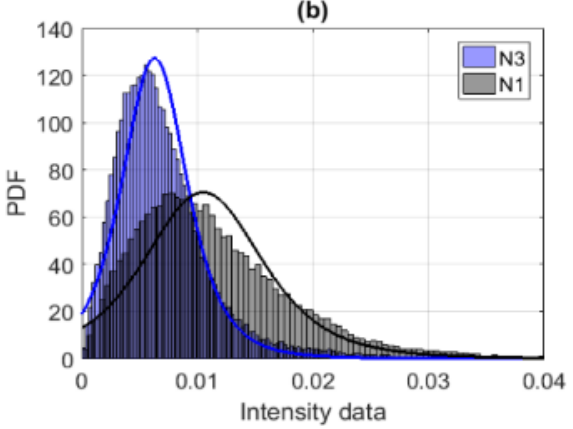

(d)

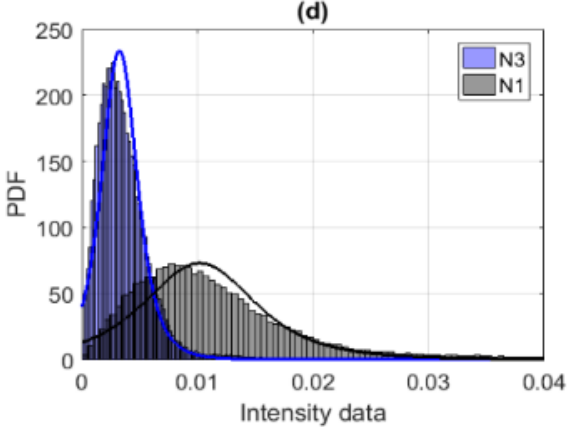

(f)

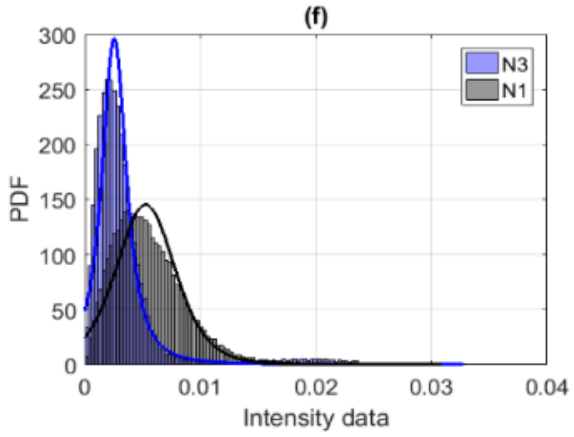

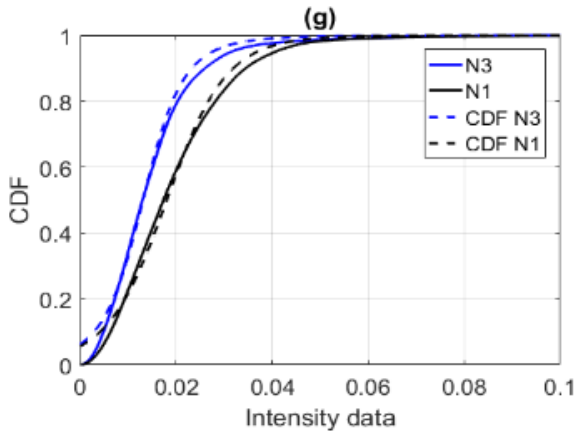

(h)

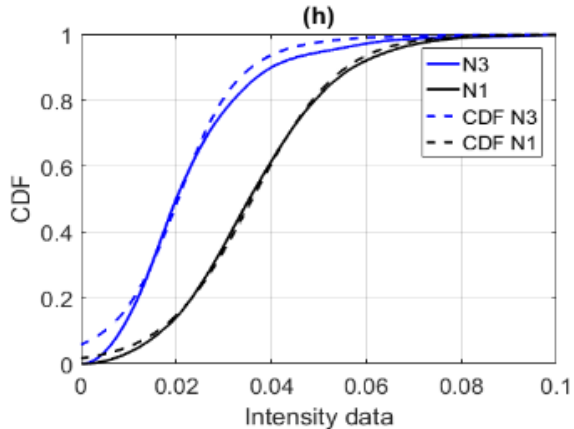

(i)

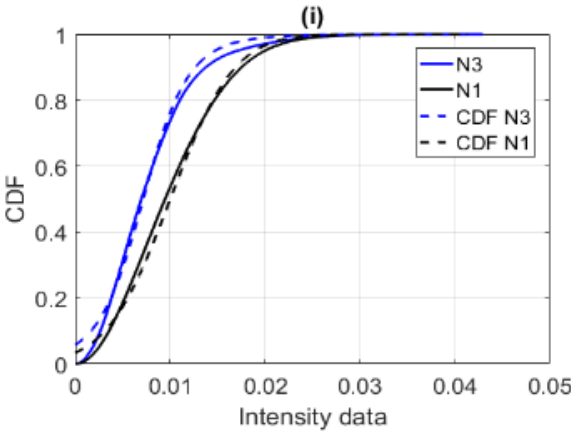

Figure 9 T-Location Scale PDF and CDF distributions fitted to experimental monostatic (N3) and bistatic (N1) data: (a) HH TUT1, (b) HH TUT2, (c) VV TUT1, (d) VV TUT2, (e) VH TUT1, (f) VH TUT2, (g) CDF HH TUT1, (h) CDF VV TUT1, and (i) CDF VH TUT1

\subsection{S-band reciprocal data}

The spectrograms for the signatures of data collected with reciprocal transmitter are shown in Figs. 1012. These were measurements for which the roles of the S-band nodes were reversed, with the transmitter node for the measurements analysed in the previous section becoming the receiver, and the other way round for the receiver node (as depicted in Fig. 1). For HH polarised data the bistatic signature of the TUT1 appears to be more intense than the corresponding monostatic, whereas the two signatures are more similar for the TUT2 even if the monostatic one appears to have a broader spread in Doppler. As observed in the previous section, negative Doppler flashes seem to be more intense than the positive Doppler flashes. For VV polarised data the monostatic signature of the TUT1 seems to be less intense than the corresponding bistatic signature, whereas the opposite situation appears to happen for the TUT2. Positive Doppler blade flashes are more intense than negative Doppler flashes, as observed in the previous section for non-reciprocal VV polarised data. The cross-polarised VH 
signatures appear to be more intense for the reciprocal data shown in Fig. 12 compared with those shown in the previous section. Bistatic signatures present fairly symmetric patterns in terms of positive vs negative baled flashes, whereas negative blade contributions are more dominant in the monostatic signatures.
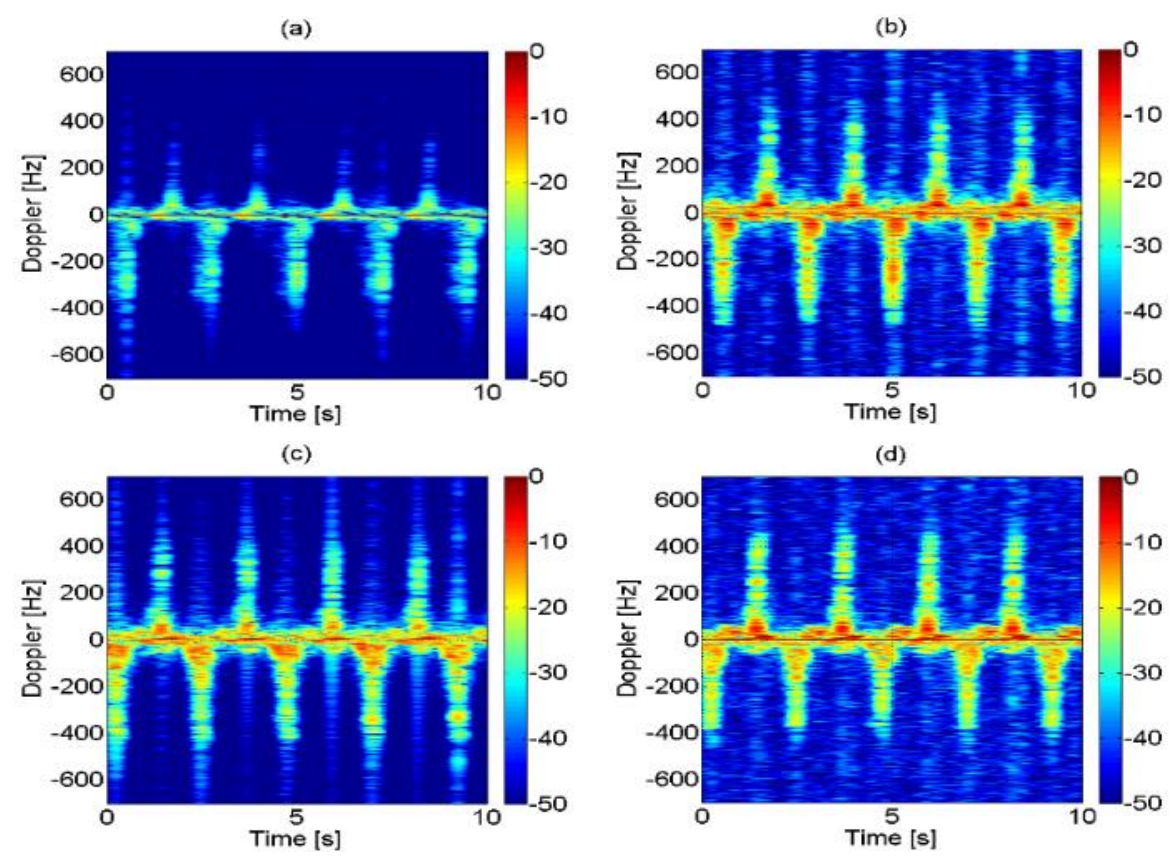

Figure 10 S-band spectrograms for HH polarised reciprocal data: TUT1 data for monostatic (a) and bistatic (b) radar node, and TUT2 data for monostatic (c) and bistatic (d) radar node
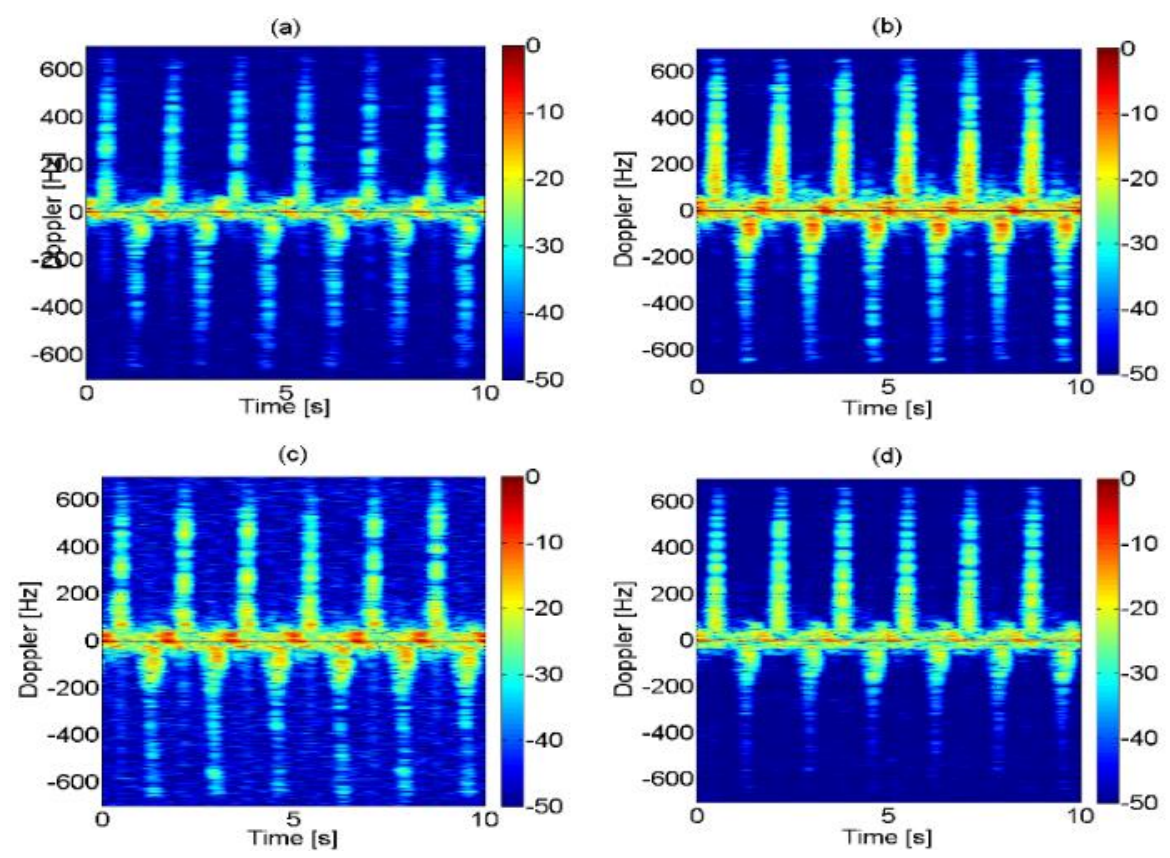

Figure 11 S-band spectrograms for VV polarised reciprocal data: TUT1 data for monostatic (a) and bistatic (b) radar node, and TUT2 data for monostatic (c) and bistatic (d) radar node 

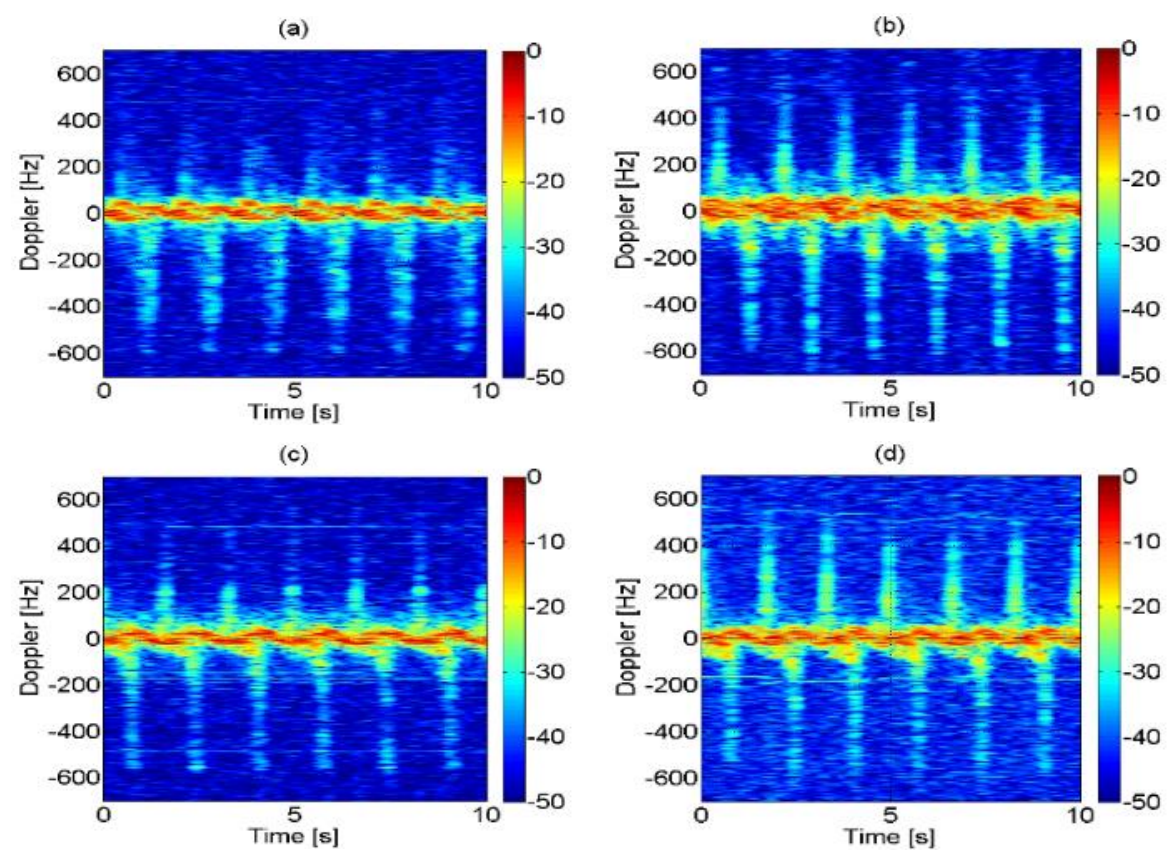

Figure 12 S-band spectrograms for VH polarised reciprocal data: TUT1 data for monostatic (a) and bistatic (b) radar node, and TUT2 data for monostatic (c) and bistatic (d) radar node

The Doppler centroid and bandwidth for the reciprocal data have also been calculated and reported in Figs. 13-15 for different polarisations and turbines under test, where 'reciprocal' means that in this case the transceiver node was the node used previously as bistatic receiver. The plots for the Doppler centroid show that the bistatic data have in general higher peak values than the corresponding monostatic, and this is more or less evident depending on the polarisation and the specific turbine, e.g. very significant for HH polarised data for the TUT1 (Fig. 13a) and not very significant for the TUT 2 (Fig. 13c). This is different from what observed in the previous section for the non-reciprocal data, where the centroid of the monostatic data appeared to have higher peaks than those in the bistatic data for the majority of plots. The bandwidth is on average higher for the monostatic data, with the exception of the VV polarised data for the TUT2 (Fig. 14d), where the opposite situation happens. This was also observed for the non-reciprocal data.

Regarding the amplitude statistics of the intensity of the radar returns, the data have been fitted to the T-Location Scale distribution as in the previous section for non-reciprocal data. Fig. 16 shows the PDF of the fitted distributions with the histograms of the corresponding data samples, for the three polarisations considered for the TUT1. It can be seen that the intensity returns recorded at Node 1 have higher mean value and longer tail than those recorded at Node 3. This situation was already observed for non-reciprocal data in the previous section, but it appears to be more significant for the data analysed here. Furthermore, it is interesting to observe that Node 1 was the monostatic transceiver for the data shown in Fig. 16, whereas it was the bistatic receiver for the previous data in Fig. 9, but in both cases the intensity returns were larger than those recorded at the other node. 
The differences between the results presented in the previous section and those referring to the 'reciprocal' measurements discussed here are interesting, especially the significant intensity increase in the cross-polarised micro-Doppler signatures and the larger differences in the amplitude statistics. The different S-band radar nodes have identical hardware for transmitter and receiver, and normal and 'reciprocal' measurements were performed only with a few minutes of temporal separation (in order to physically move the RF cables from the node to the antenna), hence more symmetry and more similar results were expected. It is thought that these noticeable differences in the radar signatures between normal and 'reciprocal' measurements are related to the different, complex EM scattering behaviour of the turbine as a function of the direction of the incident waveform from the radar transmitter and of the scattered EM field towards the radar receiver. This phenomenon can be also made more complicated by the fact that normal and 'reciprocal' data were not exactly simultaneous, so the turbine orientation in the azimuth direction (yaw angle) may have changed, contributing to the difference in signatures. At this stage, the conclusion of these preliminary observations is that the radar signatures obtained by exchanging the roles of transmitter and receiver nodes in a bistatic radar system are not symmetric and there can be significant differences. Additional work in terms of further experimental data collection and electromagnetic modelling is needed to understand how this effect is influenced by radar parameters (frequency, polarisation) and environmental conditions (wind speed, direction), and whether this can be exploited to improve radar performance (e.g. by suitable selection of the roles of different radar nodes - transmitter, receiver, or transceiver - to achieve surveillance of a certain area where wind turbines are present). 
(a)

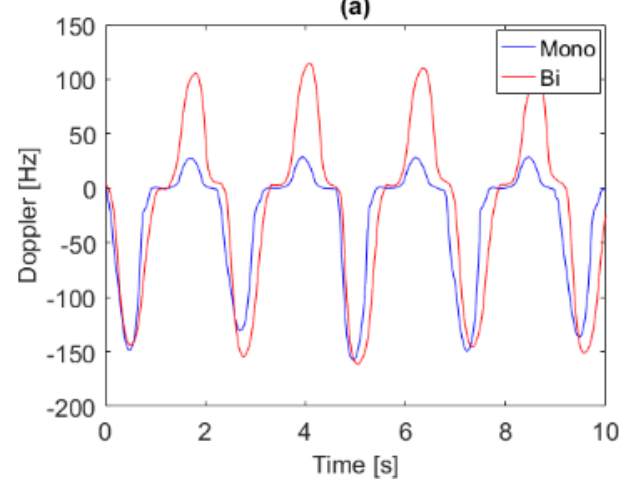

(c)

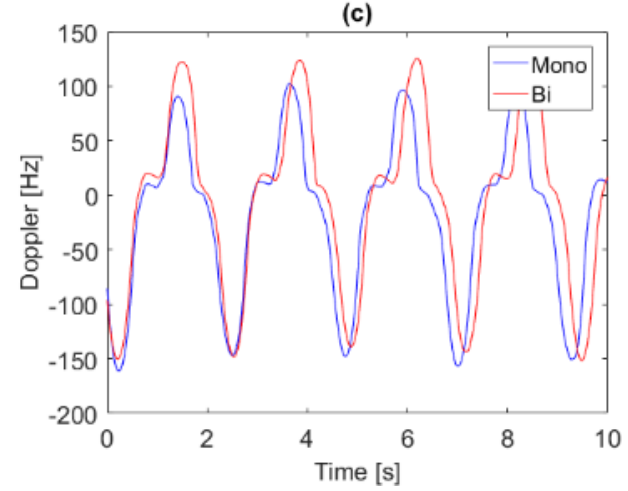

(b)

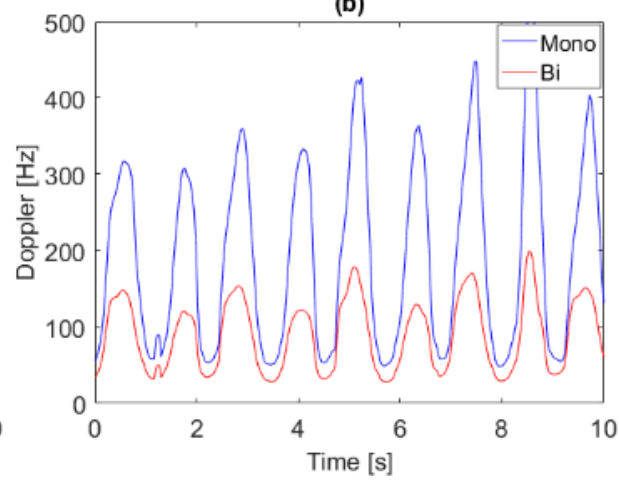

(d)

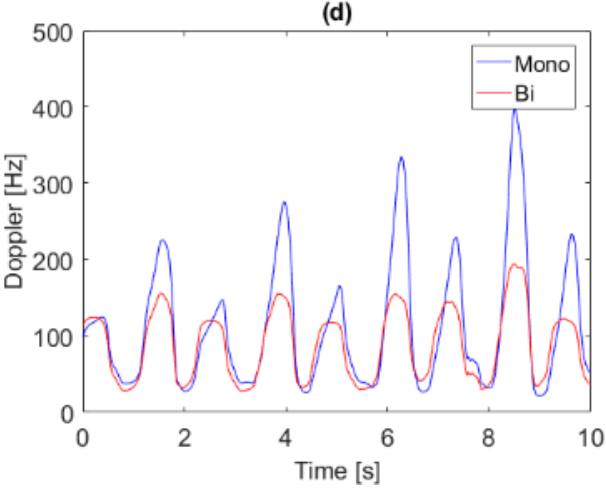

Figure 13 Monostatic vs bistatic Doppler centroid (a) and bandwidth (b) for TUT1, and Doppler centroid (c) and bandwidth (d) for TUT2 - HH polarised reciprocal S-band data
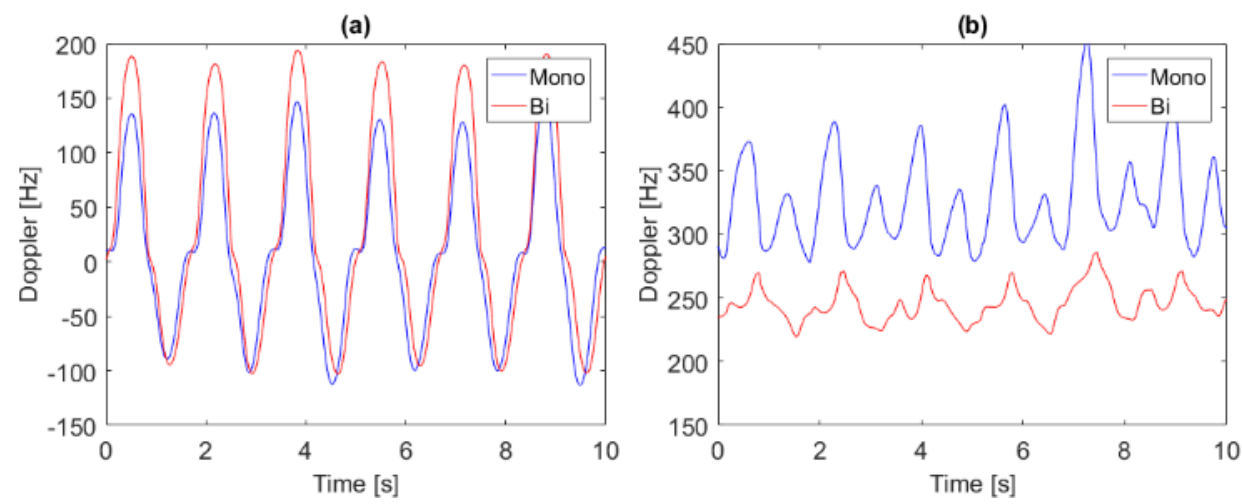

(c)
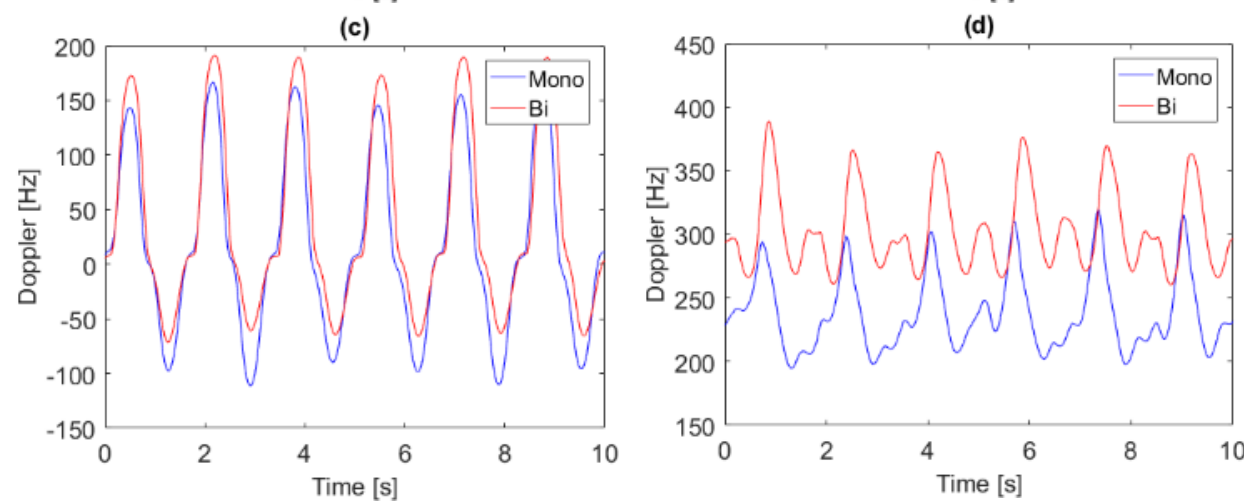

Figure 14 Monostatic vs bistatic Doppler centroid (a) and bandwidth (b) for TUT1, and Doppler centroid (c) and bandwidth (d) for TUT2 - VV polarised reciprocal S-band data 
(a)

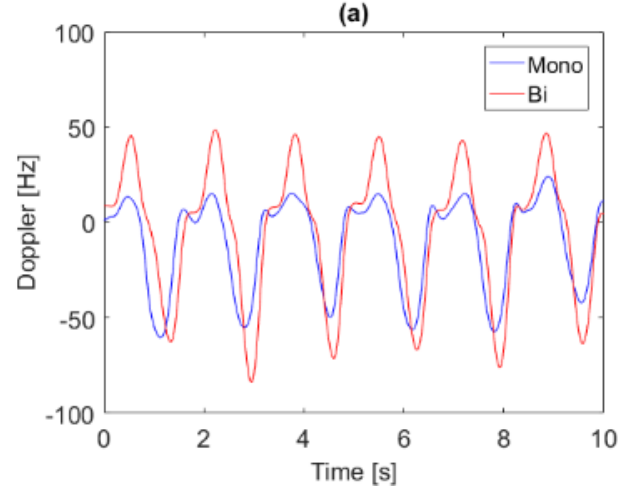

(c)

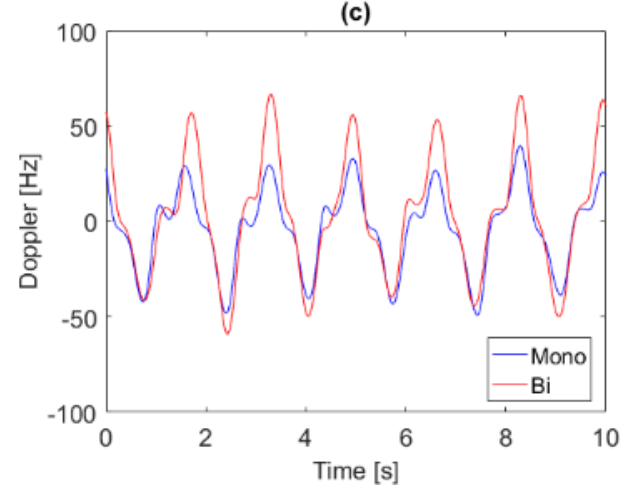

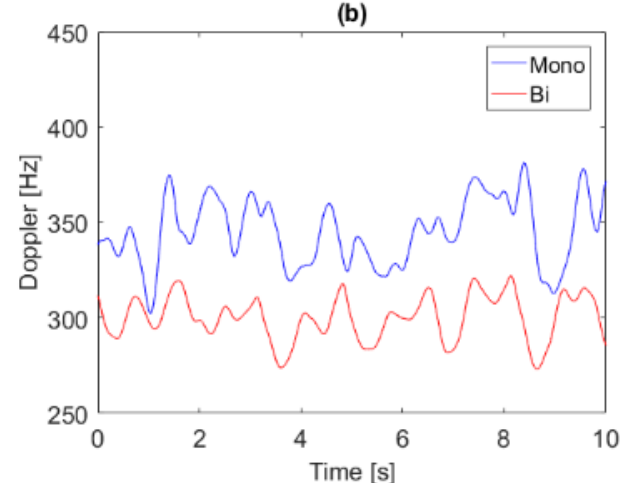

(d)

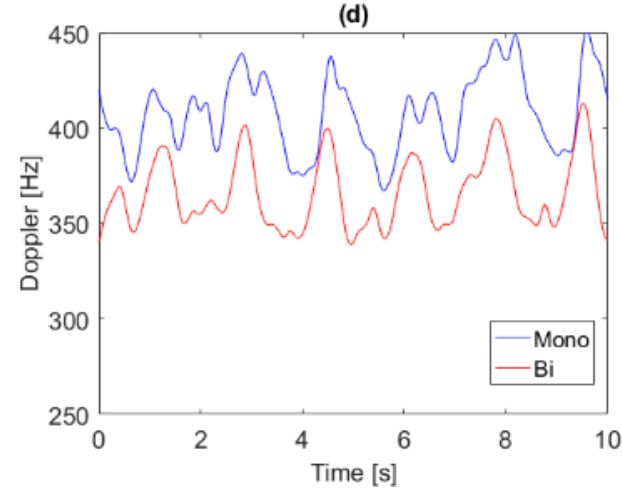

Figure 15 Monostatic vs bistatic Doppler centroid (a) and bandwidth (b) for TUT1, and Doppler centroid (c) and bandwidth (d) for TUT2 - VH polarised reciprocal S-band data
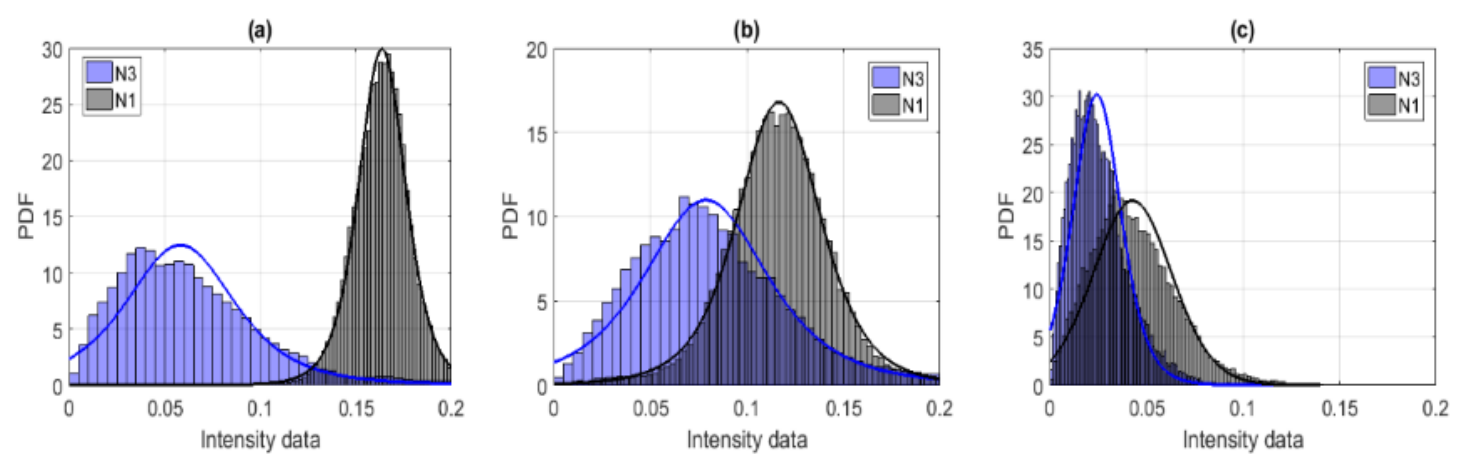

Figure 16 T-Location Scale PDF fitted to experimental monostatic (N1) and bistatic (N3) reciprocal data: (a) HH TUT1, (b) VV TUT1, and (c) VH TUT1

\subsection{X-band data}

The Doppler centroid and bandwidth for the X-band data can be seen in Fig. 17, whereas the corresponding spectrogram plots were shown in our previous work in [38] and reported here in Fig. 18 for completeness. Although not directly comparable with the S-band data where the signature of each turbine could be separated, the interest in X-band data is related to the presence of several commercial and defence radar systems for air and maritime surveillance which can be affected by wind turbine clutter. 
In general, positive Doppler blade flashes for the centroid parameter can be seen for the VV polarised data and HH polarised data, respectively in Figs. 17a and 17b, but rather weak negative Doppler blade flashes. This does not correspond with what observed for the corresponding simultaneous S-band data, where negative Doppler components could be seen, as for example in Figs. 6a and 7a. It is interesting to observe that the bandwidth parameter does not present the oscillating pattern with peaks generally observed for the S-band data, as the CW radar captures components from different turbines that rotate asynchronously, so there are multiple blade flashes with different phases. This also leads to the presence of multiple, less clean peaks in the centroid pattern for the $\mathrm{HH}$ data. On average the bandwidth parameter appears to be higher for the VV data compared with the HH data, showing that polarisation diversity can have a significant impact on the amount of collected clutter. Results for VH polarised data are not shown, as the received signal was rather weak to provide a reliable estimation of these parameters for the selected turbine under tests.

(a)

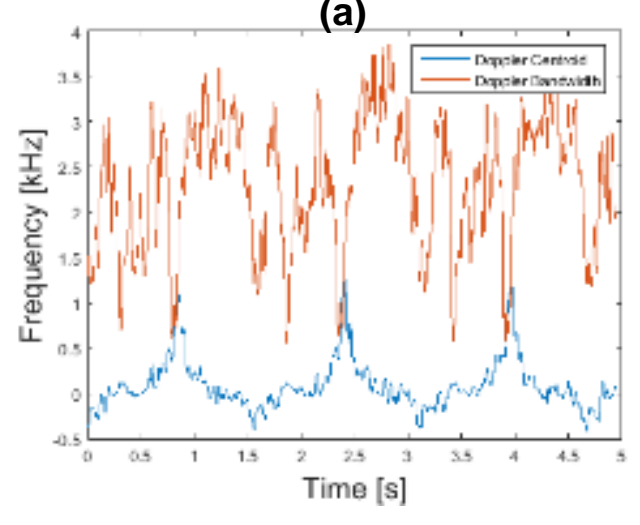

(b)

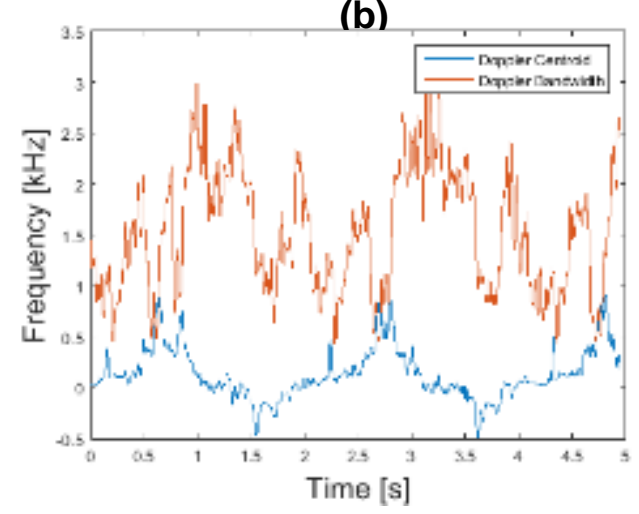

Figure 17 Doppler centroid and bandwidth for X-band data: (a) VV polarisation, and (b) HH polarisation
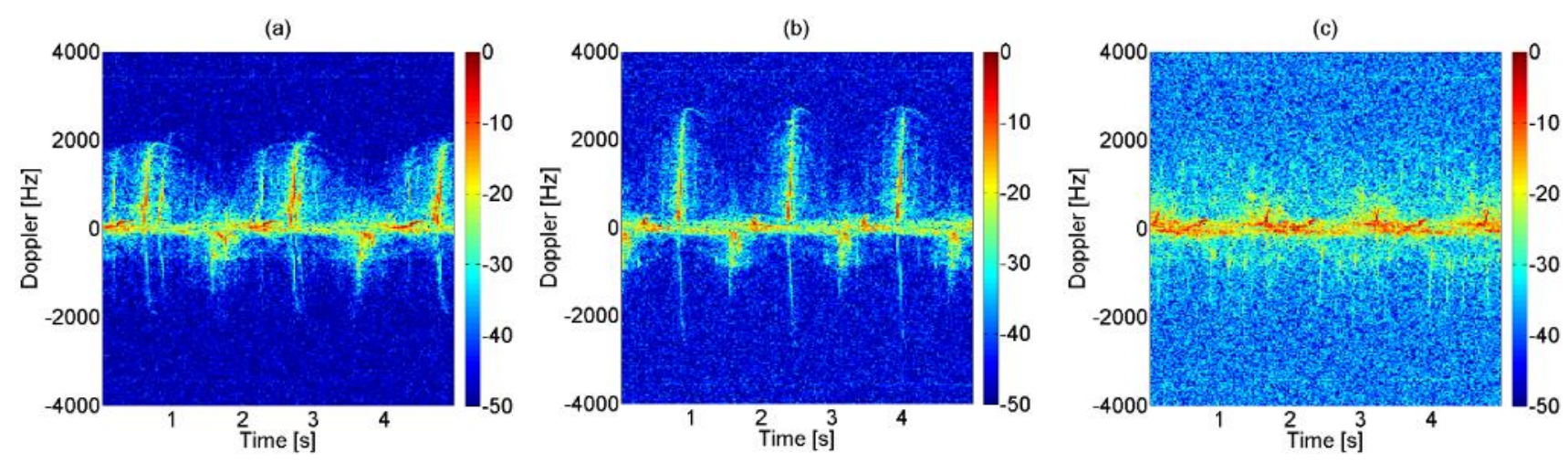

Figure 18 X-band monostatic spectrograms: (a) HH polarised data, (b) VV polarised data, and (c) VH polarised data

\section{Conclusion}

This paper presented the analysis of experimental multistatic radar data collected in January 2015 to characterise the signatures of an operational wind farm. The paper has expanded the preliminary empirical results presented in our previous conference paper [38] by extracting a series of quantitative 
parameters in order to directly compare simultaneous monostatic and bistatic signatures, such as Doppler centroid and Doppler bandwidth and amplitude statistics of the intensity returns. The data were collected using the S-band multistatic radar system NetRAD developed at UCL and an X-band monostatic radar system developed at Cranfield University, at different polarisations. These data and the related results and parameters are believed to be significant given the scarcity of experimental multistatic data of actual operational wind turbines, despite the bistatic angles being limited to $6.5^{\circ}$ and $4.65^{\circ}$ for the two turbines under test in this geometry. These results can contribute to an improved understanding and modelling of the non-stationary and fast-changing signatures of wind farm clutter, which can enhance the development of more effective mitigation solutions to reduce or eliminate its detrimental effect on the radar performance. Future solutions for the challenge of wind turbine clutter may use multistatic radar sensors networks and this understanding provides initial insight into how the clutter can vary in this multi-dimensional space.

The analysis of the quantitative parameters presented here shows that there may be significant differences between monostatic and bistatic wind turbine signatures, even at limited bistatic angles, and that the intensity and the spread in Doppler of the clutter can change with different operational conditions, such as the choice of polarisation, the turbines rotation around their yaw axis due to changing wind conditions, and the bistatic angle related to the deployment geometry of the radar nodes. In some cases, Doppler centroid peaks and average values of Doppler bandwidth appear to be higher for the monostatic S-band data compared with the simultaneous bistatic data, in other cases these differences are minimal or the opposite situation happens. At X-band the available monostatic data show that there are differences in Doppler centroid and bandwidth parameters when different polarisations, $\mathrm{HH}$ or $\mathrm{VV}$, are used.

The fact that the micro-Doppler signature at one of the radar nodes has lower values of centroid and bandwidth (i.e. it is less spread out in Doppler) could be beneficial for detecting targets of interests which may generate Doppler shifts comparable to those created by the clutter, e.g. small aircraft or helicopters. Similar comments can be made regarding the amplitude statistics of the clutter, whereby lower mean values and shorter tails of the distribution of the data collected at one radar node can be more favourable for detecting small targets. This would provide the advantage of using the data from the node (or subset of nodes) less affected by clutter to perform tasks such as target detection, tracking, and classification, whereas a radar system consisting of a single node could have its performance severely degraded in some operational conditions when the clutter signature is particularly intense at that node.

Additional data collection is needed to try and capture the variability of operational parameters such as radar frequency band, deployment geometries, and environmental conditions which affect dynamically 
the rotation speed of the blades and the orientation of the turbine with respect to the line-of-sight of the radar nodes, as well as to characterise the simultaneous monostatic and bistatic radar signatures at larger bistatic angles and when reversing the roles of transmitter and receiver of different radar nodes. As collecting this large amount of data is challenging and time consuming, the contribution of accurate computational modelling and the use of scaled models for laboratory measurements in controlled conditions is also of significant interest to complement the information from actual data.

\section{Acknowledgement}

The authors acknowledge the support of the IET A F Harvey Prize 2013 awarded to Prof. Hugh Griffiths for partial support of this work.

\section{References}

[1] “The EU's Target for Renewable Energy: 20\% by 2020”, Volume I: Report, Authority of the House of Lords, 24/10/2008.

[2] http://www.renewableuk.com/page/WindTurbines , Accessed 19/09/2016.

[3] http://www.renewableuk.com/page/Aviation, Accessed 19/09/2016.

[4] https://www.gov.uk/onshore-wind-part-of-the-uks-energy-mix\#wind-turbines-aviation-andradar, Accessed 19/09/2016.

[5] C. A. Jackson, "Windfarm characteristics and their effect on radar systems," 2007 IET International Conference on Radar Systems, pp. 1-6, 15-18 October, Edinburgh, UK.

[6] C. A. Jackson and M. M. Butler, "Options for mitigation of the effects of windfarms on radar sytems," 2007 IET International Conference on Radar Systems, pp. 1-6, 15-18 October, Edinburgh, UK.

[7] Civil Aviation Publication CAP 670, 'Air Traffic Service (ATS) Safety Requirements', available online and accessed 10/02/2016, Part C, Section 3, Appendix A, pp. 1-63.

[8] Y. F. Lok, A. Palevsky, and W. Jian, "Simulation of radar signal on wind turbine," IEEE Aerospace and Electronic Systems Magazine, vol. 26, pp. 39-42, 2011.

[9] L. R. Danoon and A. K. Brown, "Modeling methodology for computing the radar cross section and Doppler signature of wind farms," IEEE Transactions on Antennas and Propagation, vol. 61, pp. 5166-5174, 2013.

[10] O. Karabayir, S. M. Yucedag, A. F. Coskun, O. M. Yucedag, H. A. Serim, and S. Kent, "Wind turbine signal modelling approach for pulse Doppler radars and applications," IET Radar, Sonar \& Navigation, vol. 9, pp. 276-284, 2015. 
[11] F. Weinmann and J. G. Worms, "EM scattering effects caused by wind turbines," 2016 10th European Conference on Antennas and Propagation (EuCAP), Davos, 2016, pp. 1-5.

[12] C. J. Li and H. Ling, "On simulating the high-resolution radar image of a wind turbine," 2016 10th European Conference on Antennas and Propagation (EuCAP), Davos, 2016, pp. 1-3.

[13] D. de la Vega, D. Jenn, I. Angulo and D. Guerra, "Simplified characterization of Radar Cross Section of wind turbines in the air surveillance radars band," 2016 10th European Conference on Antennas and Propagation (EuCAP), Davos, 2016, pp. 1-5.

[14] P. Hamel, J. P. Adam, Y. Béniguel, G. Kubické and P. Pouliguen, "Radar cross section evaluation of a wind turbine, based on an asymptotic method," 2016 10th European Conference on Antennas and Propagation (EuCAP), Davos, 2016, pp. 1-3.

[15] F. Kong, Y. Zhang, and R. D. Palmer, "Wind turbine radar interference studies by polarimetric measurements of a scaled model," IEEE Transactions on Aerospace and Electronic Systems, vol. 49, pp. 1589-1600, 2013.

[16] A. Naqvi, Y. Shang-Te, and L. Hao, "Investigation of Doppler features from wind turbine scattering," IEEE Antennas and Wireless Propagation Letters, vol. 9, pp. 485-488, 2010.

[17] S. Chiu and P. Beaulne, "Ground moving target indication inside a wind farm: interference and mitigation," Proceedings of 10th European Conference on Synthetic Aperture Radar EUSAR, 2014, pp. 1-4, 3-5 June, Berlin, Germany.

[18] F. Darcy and D. de la Vega, "A methodology for calculating the interference of wind farm on weather radar," Antennas \& Propagation Conference LAPC 2009, pp. 665-667, 16-17 November, Loughborough, UK.

[19] R. M. Beauchamp and V. Chandrasekar, "Dual-Polarization Radar Characteristics of Wind Turbines with Ground Clutter and Precipitation," IEEE Transactions on Geoscience and Remote Sensing, vol. 54, no. 8, pp. 4833-4846, Aug. 2016.

[20] F. Kong, Y. R. Zhang and R. Palmer, "Micro-motion signatures of large wind turbines: Case study using a mobile weather radar," 2016 IEEE Radar Conference, Philadelphia, PA, 2016, pp. 1-4.

[21] O. Grande, J. Cañizo, I. Angulo, et al., "Simplified formulae for the estimation of offshore wind turbines clutter on mariner Radars," The Scientific World Journal, vol. 2014, Article ID 982508, 2014.

[22] L. Danoon, W. Al-Mashhadani and A. Brown, "Modelling the impact of offshore wind farms on safety radars onboard Oil and Gas platforms," 2016 10th European Conference on Antennas and Propagation (EuCAP), Davos, 2016, pp. 1-5.

[23] A. F. Wind, J. D. Gerber, and J. D. Griesbach, "Impacts of wind turbine farm obscurations on aircraft escort probability of success," 2014 IEEE Radar Conference, pp. 0603-0607, 19-23 May, Cincinnati, OH, USA. 
[24] C. J. Li, R. Bhalla, and L. Hao, "Investigation of the dynamic radar signatures of a vertical-axis wind turbine," IEEE Antennas and Wireless Propagation Letters, vol. 14, pp. 763-766, 2015.

[25] A. Balleri, A. Al-Armaghany, H. Griffiths, K. Tong, T. Matsuura, T. Karasudani, et al., "Measurements and analysis of the radar signature of a new wind turbine design at X-band," IET Radar, Sonar \& Navigation, vol. 7, pp. 170-177, 2013.

[26] J. Pinto, J. C. G. Matthews, and G. C. Sarno, "Stealth technology for wind turbines," IET Radar, Sonar \& Navigation, vol. 4, pp. 126-133, 2010.

[27] L. Danoon, A. El-Makadema, and A. Brown, "On the integration of lightning protection with stealth coated wind turbine blades," Wind Energy, vol. 17, pp. 1577-1585, 2014.

[28] F. Nai, S. Torres, and R. Palmer, "On the mitigation of wind turbine clutter for weather radars using range-Doppler spectral processing," IET Radar, Sonar \& Navigation, vol. 7, pp. 178-190, 2013.

[29] Gallardo-Hernando, B.; Pérez-Martínez, F.; Aguado-Encabo, F., "Detection and mitigation of wind turbine clutter in C-band meteorological radar," IET Radar, Sonar \& Navigation, vol. 4, pp. 520$527,2010$.

[30] F. Uysal, I. Selesnick, B. M. Isom, "Mitigation of Wind Turbine Clutter for Weather Radar by Signal Separation," IEEE Transactions on Geoscience and Remote Sensing, vol. 54, no. 5, pp. 29252934, May 2016.

[31] B. M. Kent, K. C. Hill, A. Buterbaugh, G. Zelinski, R. Hawley, L. Cravens, et al., "Dynamic Radar cross section and radar Doppler measurements of commercial General Electric windmill power turbines - Part 1: predicted and measured radar signatures," IEEE Antennas and Propagation Magazine, vol. 50, pp. 211-219, 2008.

[32] A. Buterbaugh, B. M. Kent, K. C. Hill, G. Zelinski, R. Hawley, L. Cravens, et al., "Dynamic radar cross section and radar Doppler measurements of commercial General Electric windmill power turbines - Part 2: predicted and measured Doppler signatures," 2007 Antenna Measurements Techniques Association (AMTA) Symposium, St Louis, MO, USA.

[33] B. M. Isom, R. D. Palmer, G. S. Secrest, R. D. Rhoton, D. Saxion, T. L. Allmon, et al., "Detailed observations of wind turbine clutter with scanning weather radars," Journal of Atmospheric and Oceanic Technology, vol. 26, pp. 894-910, 2009/05/01 2009.

[34] Chiu, S., 'Wind turbine radar clutter statistics and probability of detection', 2015 IEEE Radar Conf., Arlington, VA, May 2015, pp. 0015-0020.

[35] H. J. de Wind, K. H. Kloke, U. Böniger, H. Pratisto, 'Comparison of the recorded RCS and spectra of three different wind turbines on L- and S-band', 2015 IEEE Radar Conference, Johannesburg, 2015, pp. 266-271. 
[36] O. A. Krasnov and A. G. Yarovoy, "Polarimetric micro-Doppler characterization of wind turbines," 2016 10th European Conference on Antennas and Propagation (EuCAP), Davos, 2016, pp. $1-5$.

[37] J. M. Muñoz-Ferreras, Z. Peng, Y. Tang, R. Gómez-García, D. Liang and C. Li, "Short-Range Doppler-Radar Signatures from Industrial Wind Turbines: Theory, Simulations, and Measurements," IEEE Transactions on Instrumentation and Measurement, vol. 65, no. 9, pp. 2108-2119, Sept. 2016.

[38] F. Fioranelli, M. Ritchie, H. Griffiths and A. Balleri, "Analysis of multiband monostatic and bistatic radar signatures of wind turbines," 2015 IEEE Radar Conference, Johannesburg, 2015, pp. 277-282.

[39] F. Fioranelli, M. Ritchie, A. Balleri, H. Griffiths, "Experimental analysis of multistatic wind turbine radar clutter statistics," in Electronics Letters, vol. 52, no. 3, pp. 226-228, 242016.

[40] F. Fioranelli, M. Ritchie, A. Balleri, H. Griffiths, 'Experimental analysis of multistatic multiband radar signatures of wind turbines', IET Radar, Sonar \& Navigation, accepted in April 2016.

[41] T. E. Derham, S. Doughty, K. Woodbridge, and C. J. Baker, "Design and evaluation of a lowcost multistatic netted radar system," IET Radar, Sonar \& Navigation, vol. 1, pp. 362-368, 2007. [42] M. Ritchie, F. Fioranelli, A. Balleri, H. Griffiths, 'Measurement and analysis of multiband bistatic and monostatic radar signatures of wind turbines', Electronics Letters, 2015, 51, (14), p. 1112-1113. [43] V. C. Chen, D. Tahmoush, and W. J. Miceli, Radar Micro-Doppler Signatures: Processing and Applications, Chapter 12, pp. 345-381, Institution of Engineering and Technology, 2014. 